\title{
Evolution of the mean jet shape and dijet asymmetry distribution of an ensemble of holographic jets in strongly coupled plasma
}

\author{
Jasmine Brewer, ${ }^{a}$ Krishna Rajagopal, ${ }^{a}$ Andrey Sadofyev ${ }^{a, b}$ and Wilke van der Schee ${ }^{a, c}$ \\ ${ }^{a}$ Center for Theoretical Physics, Massachusetts Institute of Technology, \\ Cambridge, MA 02139, U.S.A. \\ ${ }^{b}$ Theoretical Division, MS B283, Los Alamos National Laboratory, \\ Los Alamos, NM 87545, U.S.A. \\ ${ }^{c}$ Institute for Theoretical Physics and Center for Extreme Matter and Emergent Phenomena, \\ Utrecht University, Leuvenlaan 4, 3584 CE Utrecht, The Netherlands \\ E-mail: jtbrewer@mit.edu, krishna@mit.edu, sadofyev@mit.edu, \\ wilke@mit.edu
}

ABSTRACT: Some of the most important experimentally accessible probes of the quarkgluon plasma (QGP) produced in heavy ion collisions come from the analysis of how the shape and energy of sprays of energetic particles produced within a cone with a specified opening angle (jets) in a hard scattering are modified by their passage through the strongly coupled, liquid, QGP. We model an ensemble of back-to-back dijets for the purpose of gaining a qualitative understanding of how the shapes of the individual jets and the asymmetry in the energy of the pairs of jets in the ensemble are modified by their passage through an expanding cooling droplet of strongly coupled plasma, in the model in a holographic gauge theory that is dual to a 4+1-dimensional black-hole spacetime that is asymptotically anti-de Sitter (AdS). We build our model by constructing an ensemble of strings in the dual gravitational description of the gauge theory. We model QCD jets in vacuum using strings whose endpoints are moving "downward" into the gravitational bulk spacetime with some fixed small angle, an angle that represents the opening angle (ratio of jet mass to jet energy) that the QCD jet would have in vacuum. Such strings must be moving through the gravitational bulk at (close to) the speed of light; they must be (close to) null. This condition does not specify the energy distribution along the string, meaning that it does not specify the shape of the jet being modeled. We study the dynamics of strings that are initially not null and show that strings with a wide range of initial conditions rapidly accelerate and become null and, as they do, develop a similar distribution of their energy density. We use this distribution of the energy density along the string, choose an ensemble of strings whose opening angles and energies are distributed as in perturbative QCD, and 
show that we can then fix one of the two model parameters such that the mean jet shape for the jets in the ensemble that we have built matches that measured in proton-proton collisions reasonably well. This is a novel way for hybridizing relevant inputs from perturbative QCD and a strongly coupled holographic gauge theory in the service of modeling jets in QGP. We send our ensemble of strings through an expanding cooling droplet of strongly coupled plasma, choosing the second model parameter so as to get a reasonable value for $R_{\mathrm{AA}}^{\mathrm{jet}}$, the suppression in the number of jets, and study how the mean jet shape and the dijet asymmetry are modified, comparing both to measurements from heavy ion collisions at the LHC.

KEYwORDS: Holography and quark-gluon plasmas, Quark-Gluon Plasma

ARXIV EPRINT: 1710.03237 


\section{Contents}

1 Introduction 1

2 String dynamics $\quad 9$

$\begin{array}{ll}2.1 & \text { The transition to null strings } \\ \end{array}$

$\begin{array}{lll}2.1 .1 & \text { Nullification in vacuum } & 16\end{array}$

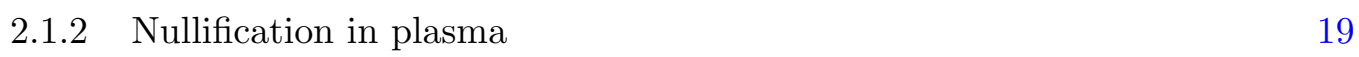

2.2 Strings with most of their energy at their endpoint 22

3 A model for an ensemble of jets in heavy ion collisions 23

4 Results, conclusions and outlook $\quad 28$

\section{Introduction}

Ultrarelativistic heavy ion collisions at the Relativistic Heavy Ion Collider (RHIC) and the Large Hadron Collider (LHC) recreate droplets of the hot matter that filled the microseconds old universe, called quark-gluon plasma (QGP). Experiments at these facilities provide unique experimental access to the properties of QGP as well as to the dynamics via which droplets of QGP form, expand and cool. These experiments have demonstrated that in the experimentally accessible range of temperatures, up to several times hotter than the crossover temperature at which cooling QGP becomes ordinary hadronic matter, droplets of QGP exhibit strong collective phenomena [1-7], with the dynamics of the rapid expansion and cooling of the initially lumpy droplets produced in the collisions successfully described by the equations of relativistic viscous hydrodynamics [8-21]. The ratio of the shear viscosity, $\eta$, to the entropy density, $s$, serves as a benchmark, because in a weakly coupled plasma, $\eta / s \propto 1 / g^{4}$ (with $g$ the gauge coupling), meaning that this ratio is large, whereas $\eta / s=1 / 4 \pi$ in the high temperature phase (conventionally called the plasma phase even though in reality it is a liquid) of any gauge theory that has a dual gravitational description in the limit of strong coupling and large number of colors [22-24]. Comparisons between hydrodynamic calculations of, and experimental measurements of, anisotropic flow in heavy ion collisions indicate that the QGP in QCD has an $\eta / s$ that is comparable to, and in particular not much larger than $1 / 4 \pi$, meaning that QGP itself is a strongly coupled liquid.

The discovery that QGP is a strongly coupled liquid at length scales of order its inverse temperature and longer even though (because QCD is asymptotically free) it consists of weakly coupled quarks and gluons when probed with high resolution challenges us to find experimental means to probe QGP at multiple length scales. The only probes that we have available are those produced in the same heavy ion collisions in which the droplets of 
QGP themselves are produced. Here we shall focus entirely on the use of high transverse momentum jets, produced at the moment of the collision in initial hard scatterings, as probes. Jets are produced with some energy and virtuality, the latter often also referred to as the jet mass. Assuming that the jet propagates in vacuum, both are (almost) conserved during the development and branching of the partonic jet shower that occurs after the jet is produced in an initial hard scattering. (Only almost because the jet may exchange soft momenta with the underlying event or with other jets.) The partonic shower develops within a cone whose opening angle is proportional to the ratio of the jet mass to the jet energy. As a partonic jet shower propagates through the strongly coupled plasma created in a heavy ion collision, however, the partons in the shower each lose energy and momentum as a consequence of their strong interactions with the plasma, creating a wake in the plasma. These interactions lead to a reduction in the jet energy (or quenching) and to modifications of the opening angle and shape of jets produced in heavy ion collisions relative to those of their counterparts produced in proton-proton collisions, that propagate in vacuum. By pursuing a large suite of jet measurements, the different LHC collaborations have observed strong modification of different jet observables in heavy ion collisions [25-50], making jets promising QGP probes. The first experimental constraints on jet quenching came from hadronic measurements at RHIC [51-53]. Analyses of jets themselves and their modification are also being performed at RHIC [54-59] and are one of the principal scientific goals of the planned sPHENIX detector [60].

A complete theoretical description of the processes by which jets are modified via passage through QGP remains challenging for the same reason that it is interesting, namely because it is a multi-scale problem. The production of jets and the processes via which an initial hard parton fragments into a shower are weakly coupled hard processes. However, the dynamics of the droplet of QGP including the wake produced in it by the passing jets and, more generally, the interaction of the jets with the QGP are sensitive to strongly coupled physics at scales of order the temperature of the QGP. One class of theoretical approaches is based upon assuming that suitably resummed weakly coupled analyses can be applied almost throughout. (See refs. [61-67] for reviews. Based on these approaches, Monte Carlo tools for analyzing jet observables are being developed [68-76] and many phenomenological studies of jets in medium have been confronted with LHC measurements of a variety of jet observables [73-75, 77-107].) However, since QGP is a strongly coupled liquid we know that physics at scales of order its temperature must be governed by strong coupling dynamics. This realization has opened the door to many connections between the physics of the QCD plasma and gauge/gravity duality [108], which yields rigorous and quantitative access to non-perturbative, strongly coupled, physics in a large family of non-abelian gauge theory plasmas that have a dual holographic description in terms of a black hole spacetime in a gravitational theory with one higher dimension. The AdS/CFT correspondence has become very successful in recent years for describing strongly-coupled dynamics in a variety of arenas. In its simplest form, AdS/CFT provides a duality between strongly coupled $\mathcal{N}=4$ supersymmetric Yang-Mills (SYM) theory in $3+1$ dimensions and classical Einstein gravity in 4+1-dimensional AdS space, or a 4+1 dimensional black hole that is asymptotically AdS in the case where the $\mathcal{N}=4 \mathrm{SYM}$ theory is at a nonzero 
temperature. Although this AdS/CFT duality has not been shown to apply to QCD, the study of the plasmas in gauge theories that do have a holographic description has led to many qualitative insights into the properties and dynamics of QGP. (See refs. [109-111] for reviews.) Within this context, there have been many interesting studies that address varied aspects of the interaction between high energy probes and strongly coupled plasma [112146]. No holographic analysis can — by itself — treat the intrinsically weakly coupled processes of jet production and fragmentation, since in all examples that are currently accessible via gauge/gravity duality the gauge theory is strongly coupled in the ultraviolet, rather than asymptotically free.

There are now two quite different phenomenological approaches being developed with the goal of addressing the multi-scale dynamics of QCD jets in strongly coupled plasma more fully, blending inputs from perturbative QCD calculations and holographic calculations where each may be relevant. The authors of refs. [147-150] have developed a hybrid strong/weak coupling model in which perturbative QCD parton showers taken from PYTHIA are modified, parton-by-parton, upon assuming that the interaction between each parton formed in the shower and the QGP follows the rate of energy loss of an energetic quark in strongly coupled plasma obtained via the holographic calculations in refs. [141, 143]. They have confronted their hybrid model with various suites of experimental data and in so doing have obtained qualitative insights into the implications of measurements of jet suppression, jet shapes, jet fragmentation functions, and the suppression, energy asymmetry and angular distributions of dijets, gamma-jets and Z-jets for parton energy loss, transverse momentum broadening, the degree to which the wakes left in the plasma by passing jets have time to equilibrate, and the resolving power of QGP.

The second approach, which we shall further develop here, was introduced by three of us in ref. [145] and is more ambitious in its use of holography, as we model each jet in its entirety as an energetic massless quark plowing through the plasma of $\mathcal{N}=4 \mathrm{SYM}$ theory. In holography, the dynamics of quarks in the fundamental representation is studied by adding space-filling D7 branes to the bulk spacetime. Open strings can end anywhere within a D7 branes, and are dual to a quark-antiquark pair in the dual boundary CFT [151]. These open strings can be constructed in many different kinds of configurations and have been used to model varied dynamical phenomena. As we shall discuss at greater length below, a pair of light quark jets in plasma is described by an open fundamental string whose endpoints shoot away from each other and the same time fall "downwards" into the black hole in the additional dimension in the AdS spacetime, with the downward angle of their motion representing (i.e. being proportional to) the opening angle of the jet in the gauge theory. One way of looking at the approach to modeling jets introduced in ref. [145] is that we seek to use inputs from perturbative QCD that are in a sense minimal, namely only those inputs that describe jet production. The way we do this is to construct an ensemble of holographic jets with an initial probability distribution for their energy and opening angle taken from perturbative QCD so as to reproduce this distribution as in proton-proton collisions. The qualitative insight obtained in ref. [145] is that even though every jet in the ensemble widens as it propagates through the strongly coupled plasma, after passage through the plasma jets with a given energy in the ensemble can have a smaller mean 
opening angle than jets with that energy would have had if they were in vacuum. This happens because there are far fewer jets with higher energies than with lower energies in the distribution (before quenching the distribution is $\sim E_{\text {jet }}^{-6}$ ) and because those jets that are initially wider lose more energy, meaning that the jets that remain with any specified energy are those narrow jets which suffered the least energy loss. This result highlights the importance of analyzing an ensemble of jets if one wishes to make comparisons, even qualitative comparisons, to jet phenomenology: because different jets with the same $E_{\text {jet }}$ that traverse the same plasma but that start out with different initial opening angles lose very different amounts of energy, it is insufficient and in fact quite misleading to attempt to draw phenomenological conclusions by looking just at single average jet with some given energy. This conclusion applies for very similar reasons in perturbative [98], holographic [145], and hybrid [149] calculations.

In the present study, which we reported on preliminarily in ref. [146], we extend the model of ref. [145] in two important ways. First, we analyze the shape of the jets in the ensemble, rather than just their opening angle. This forces us to consider the initial distribution of energy along the string more carefully. Our goal is to choose this distribution so as to reproduce the shape of jets in vacuum, and then to study how this shape is modified by passage through the plasma. In section 2 we shall find a rather remarkable way of using quite nontrivial string dynamics in the holographic gauge theory to construct an ensemble of strings (in section 3) whose mean jet shape does indeed reproduce the mean shape of QCD jets produced in proton-proton collisions. Second, we choose an ensemble of backto-back dijets with the distribution of the energy asymmetry between the two jets in an event chosen to match that measured in proton-proton collisions and analyze how this dijet asymmetry distribution is modified by passage through the plasma.

With the goal of making this paper more self-contained, we shall spend the remainder of this introduction reviewing some aspects of various previous holographic calculations that provide the basis and context for how we (and others) use selected strings in a holographic gauge theory as models for jets. Along the way we shall also set up key elements of our analysis that follows. Fig. 1, adapted from ref. [143], provides a good starting point as it illustrates many key features of how to build a holographic model for jets in plasma. The depth of the black hole horizon in the AdS direction is $1 /(\pi T)$; it sets the inverse temperature of the strongly coupled plasma in the field theory. If the figure had been drawn in vacuum, there would be no horizon and the blue geodesics would all be straight lines. The blue geodesics in the figure as drawn curve downward because of the presence of the horizon, which is to say because of the presence of the plasma. The essence of the holographic dictionary is that a depth $z$ into the bulk corresponds to a length-scale $z$ in the gauge theory. Hence, if a (red) bit of energy propagates along a (blue) trajectory that is a straight line heading rightward and downward with some angle $\sigma$ in the figure, this bit of energy is holographically dual to energy in the gauge theory that expands in size linearly in time as it propagates rightward. This is to say it is dual to a flux of energy in the gauge theory that fills a cone with an opening angle proportional to $\sigma$. In order to model a jet in vacuum, with some unchanging value of its jet mass, we must therefore find a string whose endpoint travels with some constant downward angle $\sigma_{0}$. That is, in vacuum the 


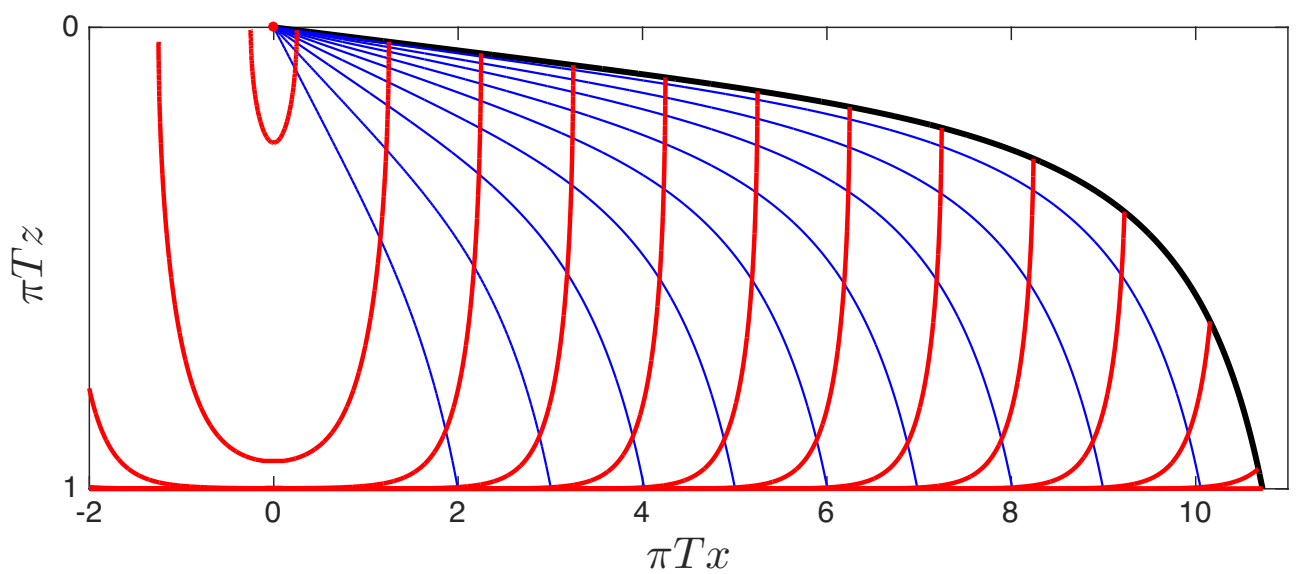

Figure 1. A null string (red) used to model a jet moving in the $x$-direction shown at several coordinate times $t$ [143]. The string starts off at the point $x=0$ on the boundary and expands at the speed of light while falling downwards into the holographic $z$-direction, towards the horizon located at $z=z_{h}=1 /(\pi T)$. The blue curves represent the null geodesics that each (red) bit of energy that makes up the string follows; the black curve is the endpoint trajectory. Different blue curves are parametrized by different values of $\sigma$, where $\sigma$ is the initial downward angle in the $(x, z)$ plane. The endpoint is the trajectory with $\sigma=\sigma_{0}$; the figure is drawn with $\sigma_{0}=0.025$. If the string were in vacuum, there would be no horizon, the blue null geodesics would all be straight lines, and the red string would maintain its initially semi-circular shape forever [128]. The opening angle of the jet which such a vacuum string represents is proportional to $\sigma_{0}$. Due to the presence of the horizon, which is to say due to the presence of the strongly coupled plasma, a blue (or black) trajectory with a given $\sigma$ curves downward: its angle in the $(x, z)$ plane, which starts out equal to $\sigma$, steadily increases. Consequently, the opening angle of a jet increases as it propagates through the plasma [143]. The energy lost from the jet to the plasma corresponds to energy density along the string traveling along blue geodesics falling into the horizon. Clearly, geodesics with smaller $\sigma$, i.e. with smaller initial angle, propagate the farther before reaching the horizon. The thermalization length of the jet, $x_{\text {therm }}$, is the distance that the endpoint travels before it reaches the horizon. It is apparent from the figure that jets with a smaller $\sigma_{0}$, meaning a narrower initial opening angle, lose their energy more slowly and have a longer $x_{\text {therm }}$ [143]. Figure adapted from ref. [143].

downward angle of the string endpoint, $\sigma_{0}$, in the gravitational description is proportional to the opening angle of the "jet" in the $\mathcal{N}=4$ SYM theory that we use to model a jet with that ratio of jet mass to jet energy in QCD. With this correspondence established, we can now read many qualitative features of jet quenching in a strongly coupled gauge theory directly from the figure. The fact that string energy traveling along blue geodesics falls into the black hole in the gravitational description is equivalent to the fact that energy is lost by the jet as the jet excites a wake in the plasma. The fact that the string endpoint trajectory, like any of the blue trajectories, curves downward corresponds to the fact that the opening angle of the jet expands as the jet propagates through the plasma, losing energy. The fact that trajectories whose initial downward angle is smaller go farther before falling into the horizon corresponds to the fact that jets whose initial opening angle, proportional to $\sigma_{0}$, is smaller lose energy more slowly, over a longer distance, and travel farther through the plasma before thermalizing in the plasma. 
Via the calculation that corresponds to the blue geodesics in figure 1 falling into the horizon, the authors of refs. [141, 143] obtained an analytic expression for the rate at which these jets, or better to say these models for jets constructed in strongly coupled $\mathcal{N}=4$ SYM theory, lose energy as they propagate through the strongly coupled plasma:

$$
\frac{1}{E_{\text {init }}} \frac{d E_{\text {jet }}}{d x}=-\frac{4 x^{2}}{\pi x_{\text {therm }}^{2} \sqrt{x_{\text {therm }}^{2}-x^{2}}} .
$$

where $E_{\text {init }}$ is the initial energy of the jet represented by the null string in figure 1 and where $x_{\text {therm }}$ is the thermalization distance of the jet, namely the distance that the string endpoint travels before falling into the horizon. This distance is related to the initial downward angle of the string endpoint, $\sigma_{0}$, by [143]

$$
T x_{\text {therm }}=\frac{\Gamma\left(\frac{1}{4}\right)^{2}}{4 \pi^{3 / 2}} \frac{1}{\sqrt{\sigma_{0}}},
$$

which quantifies the fact that jets whose opening angle is initially smaller travel farther through the plasma. For a jet that travels a small distance and loses only a small fraction of its initial energy, we can expand and integrate (1.1), obtaining [143]

$$
\frac{d E_{\text {jet }}}{d x}=-E_{\text {jet }} \frac{256 \pi^{7 / 2}}{\Gamma\left(\frac{1}{4}\right)^{6}} T^{3} x^{2} \sigma_{0}^{3 / 2}+\mathcal{O}\left(x^{4}\right) .
$$

When we construct our ensemble of strings with which we shall model an ensemble of jets in plasma in section 3, we shall follow ref. [145] in treating the proportionality constant between the initial downward angle of the endpoint of a string, $\sigma_{0}$, and the opening angle of the jet that we wish to model with that string as a free parameter. This is the first of two free parameters in our model; we shall denote it by $a$ and will define it precisely in section 3.

It is important to realize that not all string configurations that can be constructed in the gravitational dual of $\mathcal{N}=4 \mathrm{SYM}$ theory behave like the string in figure 1 . Not all by any means. Strings have a nonzero tension, but in figure 1 the string tension does not affect the dynamics of the red string because each bit of string is following a null geodesic. One can (and in fact we will in the next section) instead construct string configurations with different initial conditions in which the string worldsheet is, at least initially, not null and in which the string tension affects the dynamics of the string to such a degree that the downward angle at which the endpoint of the string moves changes substantially as it propagates even in vacuum. It is not immediately apparent how such a string can serve as a model for a jet, since on the face of it would seem to correspond to a jet whose virtuality changes substantially after the jet has been created, something that does not happen in QCD since a high energy jet once formed interacts at most softly with other jets or with the underlying event, meaning that by momentum conservation the virtuality of a jet in QCD hardly changes. In the gravitational description of strongly coupled $\mathcal{N}=4 \mathrm{SYM}$ theory, though, there are (non-null) strings in vacuum in which an end of the string feels a force from the rest of the string that changes its trajectory. This means that in strongly coupled 
$\mathcal{N}=4$ SYM theory there are configurations in which a flux of energy behaves completely differently from a jet in QCD. (This is unsurprising: most string configurations in the gravitational dual do not correspond to anything that looks like a jet. And, furthermore, in strongly coupled $\mathcal{N}=4 \mathrm{SYM}$ theory hard processes do not produce jets [124, 126].) It remains an open question whether a subset of strings whose end point trajectories change their downward angle can nevertheless be used directly as models for jets; investigating this would require computing the gauge theory energy flux and looking for instances where virtuality and opening angle do not change, even when the string end point trajectory does. In the present work, we follow a more straightforward approach. We choose to model jets in QCD by choosing strings in the gravitational description of strongly coupled $\mathcal{N}=4$ SYM theory whose endpoints follow a trajectory with some constant downward angle $\sigma_{0}$ in vacuum, curving downward only because of the presence of the black hole horizon, modeling jets whose opening angles change only because they are propagating through plasma. The simplest way that we know of choosing strings that constitute good models for jets in QCD is to choose null strings, as in figure 1, following an approach that goes back to ref. [128].

Our discussion to this point has left the distribution of energy along the string unspecified. For null strings in vacuum, whatever distribution of energy along the string we choose initially (respecting open string boundary conditions) will simply propagate unchanged along the blue null geodesics (which are straight in vacuum). Since a null string like that in figure 1 propagates for an initial period of time $\ll 1 /(\pi T)$ as if it were in vacuum, we have considerable freedom in choosing the initial energy density along the string. After the string has propagated through the plasma for a distance that is $\gg 1 /(\pi T)$, its shape is no longer semicircular, as in vacuum. In fact, after propagation through the plasma it takes on the shape of a segment of the string that describes an infinitely heavy quark being dragged through the plasma [143], a shape that was first worked out in refs. [112, 114, 115]. As the string propagates through the plasma over a distance $\gg 1 /(\pi T)$ and blue trajectory after blue trajectory peels away and falls into the horizon, eventually the only aspect of the initial distribution of energy along the string that matters is the energy that is initially very close to the endpoint of the string. For this reason, the authors of ref. [143] chose an initial distribution of energy along the string that takes the near-endpoint form $\propto 1 /\left(\sigma^{2} \sqrt{\sigma-\sigma_{0}}\right)$ dictated by the open string boundary conditions everywhere along the string. Although operationally reasonable, the logic behind this choice is not fully satisfactory since it is based upon using the form of the string energy density after the string has propagated for a long distance through the plasma to choose the distribution of energy along the string initially, when the string is still behaving as if it were in vacuum. It would be better to have an argument based upon the physics of strings in vacuum for choosing the initial distribution of energy along the string. We shall remedy this lacuna in section 2 .

In section 2 we study the dynamics of strings that are initially not null. As anticipated, in vacuum their endpoints do not follow trajectories with a constant downward angle, meaning that they (initially) represent objects whose virtuality is not obviously conserved which makes it unclear how they can be used to model jets in QCD. As an extreme example, extreme in the sense that they are the least apparently jet-like of any of the strings we analyze, we include strings similar to the ones considered in ref. [139] in which 
the downward angle of the string endpoint changes suddenly. From our analysis of their dynamics, however, we find that a large class of strings that are initially not null become null strings as they fall in the AdS vacuum: after a certain "nullification time" that we compute, every bit of string moves along a null geodesic, as anticipated in refs. [141, 143]. After nullification, the string endpoints follow trajectories with a constant downward angle meaning that after nullification all these strings end up becoming jet-like. And, quite remarkably, we find that for a rather diverse set of initial conditions for the energy density along the string, as long as we don't make the string null initially (in which case the energy distribution would not change in vacuum) after the string nullifies the distribution of energy density along the string has evolved such that it is approximated by a scaling form parametrized only by the downward angle of the string endpoint after nullification. Near the string endpoint this scaling form agrees with the expression for the distribution of energy density along the string obtained from the near-endpoint expansion of refs. [141, 143], as it must. We show that in plasma, which is to say when the gravitational description of the physics includes a horizon, the strings that we analyze nullify while they are still far above the horizon meaning that their nullification occurs as it would in vacuum.

The results for the dynamics of strings that are initially not null, in section 2 , motivate our construction, in section 3, of an ensemble of null strings as a model for an ensemble of jets. We choose the distribution of energy along a null string in this ensemble with a specified initial downward angle $\sigma_{0}$ according to the scaling form for this distribution, namely the scaling form attained by initially non-null strings. This means that we are using nontrivial string dynamics in (the dual of) strongly coupled $\mathcal{N}=4 \mathrm{SYM}$ theory, dynamics that does not itself appear to be jet-like, to determine how to distribute the energy density along the strings in the ensemble of null strings that we subsequently use to model an ensemble of jets. Another way of describing this is that among all the possible null strings that can be constructed in the dual of $\mathcal{N}=4$ SYM theory, the subset that we choose to use in our ensemble of strings are those with a particular scaling form for their energy distribution such that they can be formed either by starting with null strings from the beginning or by starting with strings that are initially not null and evolving them until they nullify.

As in ref. [145], we choose the distribution of the initial jet energies and opening angles for the jets in our ensemble from perturbative QCD calculations as appropriate for QCD jets in proton-proton collisions. For each jet, we choose the initial distribution of energy along the string that represents that jet in our model according to the scaling form obtained via our holographic analysis of the nullification of strings. This specifies the jet shape for each jet in our ensemble. Remarkably given that the strongly coupled dynamics by which the strings nullify has no apparent analogue in QCD, we find in section 4 that, upon fitting the single parameter $a$, our model yields a very good description of the mean jet shape in $\mathrm{QCD}$, as measured in proton-proton collisions by the CMS collaboration [33].

With our ensemble of strings fully specified by using the distribution of energy along each string taken from the scaling form obtained via our holographic analysis of nullification, in section 4 we send the ensemble through an expanding cooling droplet of hydrodynamic fluid (described in section 3). In so doing, we introduce a second free parameter 
in the model, which we denote by $b$, which is the proportionality constant between the temperature of the QCD plasma that we are modeling and the temperature of the $\mathcal{N}=4$ SYM plasma (with more degrees of freedom) that we are using as a model. We choose $b$ such that the modification in the number of jets with a given energy in the ensemble after it has passed through the droplet of plasma relative to that in the initial ensemble is comparable to that seen in data. We then compute the modification to the mean jet shape, and compare to experimental data [33]. We find a narrowing in the jet shape at small angles that is comparable to that seen in data, but because we are not including the contribution to reconstructed jets coming from the wake in the plasma our model cannot describe the modification to the mean jet shape at larger angles. We then consider an ensemble of dijets, with the dijet asymmetry distribution chosen to reproduce that measured in proton-proton collisions, and compute the modification to this distribution caused by passage through the droplet of plasma. Here again we compare to experimental data from heavy ion collisions at the LHC [27]. We close with a look ahead at possible future improvements to the model. Our results for the modification of the dijet asymmetry distribution are promising but they are not in quantitative agreement with the data; this motivates a future analysis of an ensemble of trijets, since in reality (and unlike in our model dijet ensemble) much of the dijet asymmetry seen in proton-proton collisions comes from events in which there is a third jet present.

In brief summary, we present in this work a model for jet quenching in holography in which we represent each jet in an ensemble as a null string which propagates through an expanding cooling droplet of strongly coupled plasma. In section 2 we study the nullification of strings in holography that are initially not null, and find a scaling form for the distribution of energy along a nullified string. We specify the single parameter needed to fix this distribution by fitting to CMS data on the vacuum jet shape in section 3 . With the vacuum shape of jets in the holographic model fixed to be similar to CMS data on the jet shape in proton-proton collisions, the only input to specify the shape of a jet is its opening angle. Starting from an ensemble of jets with energy and opening angle distributions taken from perturbative QCD calculations, in section 4 we calculate the modifications of the jet shape and dijet asymmetry of this ensemble after each jet propagates through the strongly coupled plasma.

\section{String dynamics}

With the aim of studying the dynamics and evolution of an ensemble of null strings in $\mathcal{N}=4$ SYM plasma as a model for an ensemble of jets, and in particular for the purpose of choosing the shape of the distribution of energy density along the null strings, we begin with a study of strings in 4+1-dimensional AdS space that are, initially, not null. Within the context of this paper, the purpose of this section is to provide a scaling form for the distribution of energy density along a string that is initially not null after it has nullified (Figure 5). We would like to emphasize in addition, however, that to our knowledge this section presents the first analysis of the string nullification process. This makes this section of interest in a context that is broader than our model, as it provides a setting in which 
to understand the previous use of null strings as models for jets in the literature as well as describing how non-null strings used in a variety of contexts evolve to become null.

In holography, a pair of light quarks is represented by an open fundamental string in AdS [151]. The 5-dimensional metric in AdS which corresponds to a constant-temperature plasma in the 4-dimensional $\mathcal{N}=4 \mathrm{SYM}$ theory on its boundary is

$$
\mathrm{d} s^{2}=\frac{L^{2}}{z^{2}}\left(-f(z) \mathrm{d} t^{2}+\mathrm{d} \vec{x}_{\perp}^{2}+\mathrm{d} y^{2}+\frac{\mathrm{d} z^{2}}{f(z)}\right),
$$

where $z$ is the additional direction in AdS space, $f(z)=1-z^{4} / z_{h}^{4}$, and the black hole is located at $z=z_{h} \equiv 1 / \pi T$. Here $\vec{x}_{\perp}$ and $y$ are field theory coordinates specifying the transverse plane and the beam direction, respectively. This metric is an exact solution to Einstein's equations for a constant-temperature plasma. We shall later (in section 3 ) choose a temperature $T$ that varies in space and time so as to model an expanding cooling droplet of plasma but for a spatially-varying temperature profile this model neglects transverse flow, fluid viscosity, and gradients.

The dynamics of strings in this geometry are most conveniently solved numerically using the Polyakov action (see refs. [128, 129, 152]):

$$
S_{P}=-\frac{T_{0}}{2} \int d \tau_{w s} d \sigma_{w s} \sqrt{-\eta} \eta^{a b} \partial_{a} X^{\mu} \partial_{b} X^{\nu} G_{\mu \nu}
$$

with $T_{0}=\sqrt{\lambda} / 2 \pi$ the string tension with $\lambda$ the 't Hooft coupling, $\tau_{w s}, \sigma_{w s}$ the string worldsheet coordinates, $G_{\mu \nu}$ the bulk AdS metric and $\eta_{a b}$ the string worldsheet metric, which determines the gauge choice in mapping $\tau_{w s}$ and $\sigma_{w s}$ coordinates to target space coordinates $X^{\mu}$. The gauge degree of freedom $\eta_{a b}$ can be solved for by varying the action, giving the constraint equation:

$$
\gamma_{a b}=\frac{1}{2} \eta_{a b} \eta^{c d} \gamma_{c d}
$$

where $\gamma_{a b}=\partial_{a} X \cdot \partial_{b} X$ is the induced metric on the string worldsheet. Since the worldsheet metric is a gauge choice, the functions $X^{\mu}\left(\tau_{w s}, \sigma_{w s}\right)$ can be chosen to make the numerics more straightforward. Since we will typically solve the equations of motions in steps along $\tau_{w s}$, this for instance requires that different parts of the string cover the part of spacetime in a similar pace in the $\tau_{w s}$ variable. This can be done by defining [129]

$$
\eta_{a b} \equiv\left(\begin{array}{cc}
-\Sigma\left(X^{\mu}\right) & 0 \\
0 & 1 / \Sigma\left(X^{\mu}\right)
\end{array}\right),
$$

with the stretching function $\Sigma\left(X^{\mu}\right)$, which is commonly chosen to cancel singularities in the equations of motion. In all the string evolutions presented in this paper, we shall use

$$
\Sigma=\left(\frac{1-z}{1-z_{0}}\right)^{\alpha}\left(\frac{z_{0}}{z}\right)^{\beta}
$$

with $\alpha$ and $\beta$ typically 1 or 2 . From the action we can also obtain the target space energymomentum density:

$$
\pi_{\mu}^{a}\left(\tau_{w s}, \sigma_{w s}\right)=\frac{1}{\sqrt{-\eta}} \frac{\delta S_{\mathrm{P}}}{\delta\left(\partial_{a} X^{\mu}\left(\tau_{w s}, \sigma_{w s}\right)\right)}=-T_{0} \eta^{a b} \partial_{b} X^{\nu} G_{\mu \nu}
$$


The energy-momentum density at some time $t$ is then given by

$$
p_{\mu}\left(t, \sigma_{w s}\right)=\frac{\sqrt{\lambda}}{2 \pi} \sqrt{-\eta}\left(\pi_{\mu}^{\tau}\left(t, \sigma_{w s}\right)-\pi_{\mu}^{\sigma}\left(t, \sigma_{w s}\right) \frac{\partial_{\sigma_{w s}} t}{\partial_{\tau_{w s}} t}\right) .
$$

We shall analyze the string dynamics throughout in classical Einstein gravity and using classical equations of motion, which means everything is done in the limit of strong coupling. In the strict $\lambda \rightarrow \infty$ limit there are no quasi-particles and hence also no distinguishable quark-antiquark pairs. In AdS this is dual to the statement that in this strict limit creating a string requires an infinite energy of order $\mathcal{O}(\sqrt{\lambda})$. Our limit hence has to interpreted as a large but finite coupling, where it does make sense to consider a quark-antiquark pair, dual to a string. Later, when we quote numerical results we shall always take $\lambda=5.5$, as in ref. [153].

We create each string at a single point $\left(z_{0}, t_{0}, x_{0}\right)$ in the AdS spacetime. Without loss of generality we can set $x_{0}=0$ and $t_{0}=0$. One way of varying the initial conditions for our strings is to vary $z_{0}$. We must also specify initial conditions for the velocity of the string in the AdS spacetime as a function of the string worldsheet parameter $\sigma_{w s}$, subject to open string boundary conditions. The aim of this section is to introduce several classes of initial conditions for the string and to show that when we choose the string to not be null initially its dynamics turn it into a null string (a string where each segment travels along an independent null geodesic) after a period of time, which we compute. We find it striking that, although the nullification process occurs through strongly-coupled dynamics which may have no direct analog in the dynamics of jets in QCD, it yields an approximate scaling form for the distribution of energy density along the string after the string nullifies. We shall use this scaling form in sections 3 and 4 when we follow the evolution of an ensemble of null strings, which serve as models for jets, as they pass through an expanding and cooling droplet of plasma.

We shall analyze six classes of initial conditions for the strings, depicted in figure 2 . The initial velocity of the string in the $x$-direction is given as a function of $\sigma_{w s}$ by the six expressions:

$$
\begin{aligned}
& \partial_{\tau_{w s}} x\left(\sigma_{w s}\right)=A \cos \left(\sigma_{w s}\right), \\
& \partial_{\tau_{w s}} x\left(\sigma_{w s}\right)=A\left(\frac{1}{2} \tanh \left(4\left(\sigma_{w s}-\frac{\pi}{2}\right)\right)-\operatorname{sech}^{2}(2 \pi) \sin \left(2 \sigma_{w s}\right)\right) \\
& \partial_{\tau_{w s}} x\left(\sigma_{w s}\right)=A \cos \left(\sigma_{w s}\right), \\
& \partial_{\tau_{w s}} x\left(\sigma_{w s}\right)=A \cos ^{3}\left(\sigma_{w s}\right) \\
& \partial_{\tau_{w s}} x\left(\sigma_{w s}\right)=A\left(\frac{1}{2} \tanh \left(4\left(\sigma_{w s}-\frac{\pi}{2}\right)\right)-\operatorname{sech}^{2}(2 \pi) \sin \left(2 \sigma_{w s}\right)\right) \\
& \partial_{\tau_{w s}} x\left(\sigma_{w s}\right)=A\left(\sigma_{w s}-\frac{1}{10} e^{-10\left(\pi-\sigma_{w s}\right)}+\frac{e^{-10 \sigma_{w s}}}{10}-\frac{\pi}{2}\right)
\end{aligned}
$$

with $A$ a parameter that we specify as described below. The velocity in the holographic 


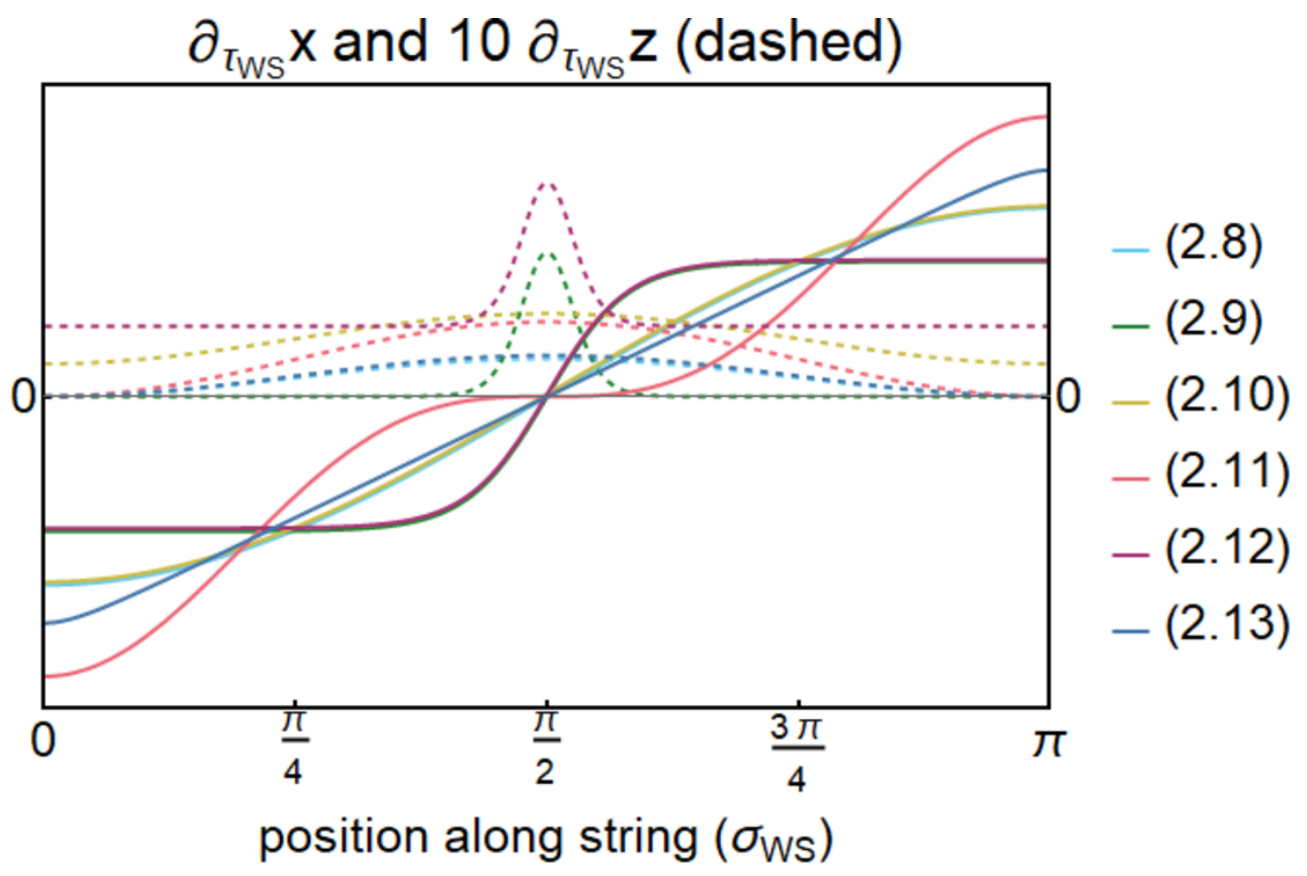

Figure 2. We show the shapes of the profiles that we use for the initial conditions on the velocities in AdS of the strings that we analyze, as given in eqs. (2.8)-(2.13) and eqs. (2.14)-(2.15). The solid curves show the initial velocity of the string in the $x$-direction as a function of the worldsheet coordinate $\sigma_{w s}$. (The string endpoints are at $\sigma_{w s}=0$ and $\pi$; the string midpoints are at $\sigma_{w s}=\pi / 2$.) The dashed curves give the initial velocity in the $z$-direction ("downward" into the bulk of AdS), enhanced by a factor of 10 for visibility. As discussed in the text, the small but nonzero $z$ velocity is useful numerically to make $\partial_{\tau_{w s}} t$ continuous at the midpoint of the string. The relationship between the worldsheet time coordinate $\tau_{w s}$ and the AdS time $t$ is given in terms of these functions in eq. (2.16).

$z$-direction is given by

$$
\partial_{\tau_{w s}} z\left(\sigma_{w s}\right)=\frac{A}{200}\left(1-\cos \left(2 \sigma_{w s}\right)\right)+A \sigma_{s}
$$

for (2.8), (2.10), (2.11) and (2.13) and by

$$
\partial_{\tau_{w s}} z\left(\sigma_{w s}\right)=\frac{A}{40}\left[\tanh \left(10\left(\sigma_{w s}-\frac{\pi}{2}\right)+\frac{1}{2}\right)+1\right]\left[\tanh \left(10\left(\frac{\pi}{2}-\sigma_{w s}\right)+\frac{1}{2}\right)+1\right]+A \sigma_{s}
$$

for (2.9) and (2.12). This $z$-velocity is small but useful to ensure that $\partial_{\tau_{w s}} t$ is continuous at $\sigma_{w s}=\pi / 2$. We shall set the parameter $\sigma_{s}=0$ in (2.8), (2.9), (2.11) and (2.13). This means that in these four classes of initial conditions, the string initially has a nonzero velocity in the $z$-direction only near $\sigma_{w s}=\pi / 2$. And, in these four classes of initial conditions the initial velocity of the string endpoints (at $\sigma_{w s}=0$ and $\pi$ ) are horizontal, corresponding initially to a collimated flow of energy with vanishing opening angle. If it were possible to create a jet with zero initial virtuality in QCD, its virtuality would remain zero; it would never fragment into a shower and would never fill a cone. No production mechanism for a 
collimated object like this is known in QCD. We shall see below, however, that in $\mathcal{N}=4$ SYM the strongly coupled dynamics ensures that an object created with zero opening angle like this does not stay that way. The strongly coupled dynamics turns this initially collimated object into something that later becomes jet-like. Which is to say that the string (which is initially not null) nullifies. In initial conditions (2.10) and (2.12) we choose a nonzero value of the parameter $\sigma_{s}$ such that the initial downward angle of the string endpoints is $0.7^{\circ}$ and $2.8^{\circ}$ respectively.

To complete the specification of the initial conditions that we shall analyze, we note that the velocity in the time direction follows from the constraint equation $\eta_{00}=0$ and from our assumption that the string starts at a single point. It is given by

$$
\partial_{\tau_{w s}} t=\sqrt{\left(\partial_{\tau_{w s}} z\left(\sigma_{w s}\right)\right)^{2}+\left(\partial_{\tau_{w s}} x\left(\sigma_{w s}\right)\right)^{2}} .
$$

Last, we describe how we choose the value of the parameter $A$. By conformal invariance we can keep one parameter fixed and then vary other parameters without loss of generality. In this section, we choose to fix $A$ such that the energy (2.7) is $E=1000$. We performed several numerical checks, verifying for all of our evolutions that the constraint equations are satisfied and that the total energy as obtained from (2.7) is conserved up to our numerical precision $\left(10^{-4}\right.$ or better).

\subsection{The transition to null strings}

At the start of the evolution of the strings with initial conditions (2.8), (2.9), (2.11) and (2.13), in which the initial downward angle of the string endpoints vanishes, the string tension is a crucial ingredient in determining the dynamics of the string. The string stretches initially, losing kinetic energy as it does so. This effect is especially strong near the boundary of the AdS spacetime, which is to say near the endpoint of the string, where the larger proper distance due to the large AdS metric factor requires the string to have a large initial energy to off-set the potential energy cost of the stretching. This suggests that there should be a sense in which strings of this type which start closer to the AdS boundary (smaller $z_{0}$ ) have larger energy. We shall make this precise below.

We shall see that what happens to these strings is that the string tension succeeds in pulling the string endpoints away from the AdS boundary and giving them a nonzero downward angle even though their initial downward angle vanishes. (This dynamics has no apparent analogue in the physics of jets in QCD.) After some time of evolution, then, the strings have fallen into the bulk AdS space and the increasing kinetic energy becomes dominant over the potential energy. At this stage the string tension no longer has a significant effect on the dynamics of the string. To a good approximation, each segment of the string travels on a null geodesic: the string nullifies. Focusing on the endpoint of the string, the string tension initially curves its trajectory downward, away from the AdS boundary, but after some time its downward angle stops increasing and it henceforth follows a null geodesic with a constant downward angle. We shall call the downward angle reached by the string endpoint as the string becomes null $\sigma_{0}$. We can now state the precise sense in which strings which start closer to the AdS boundary have larger energy: we shall 
show that, for strings whose initial downward angle vanishes, the smaller the $z_{0}$ we choose the larger the energy we must choose if we wish to end up with a specified value of $\sigma_{0}$ after nullification.

If instead of starting off with a string whose endpoint initially moves horizontally we start off with a string that is initially null, the discussion above changes completely. There is no stretching effect. The string tension never plays a significant role in the string dynamics. And, the initial downward angle of the string endpoint keeps its initial nonzero value $\sigma_{0}$ throughout. These are the strings that we shall use as models of QCD jets in vacuum. However, in this case the dynamics leaves the distribution of energy density along the string unchanged also, which gives us no clue as to what distribution to choose. The initial conditions (2.10) and (2.12) with initial downward angles for the string endpoints that are nonzero are not null, but they (in particular (2.12)) are much closer to being null than any of our initial conditions for strings whose endpoints are initially horizontal. We therefore expect that these strings should nullify more quickly, with less rearrangement of the energy density along the string. And, we expect that for these strings it need not be the case that reducing $z_{0}$ means increasing the energy needed to achieve a specified $\sigma_{0}$.

Note that since in vacuum null geodesics in AdS are just straight lines it is clear that a nullified string is fully specified in vacuum by how much energy goes downward at what angle in AdS. As we illustrated in figure 1, it is also true in plasma that a nullified string is completely specified by the initial downward angle of each bit of energy along the string. However, in this case the blue null geodesics in figure 1 curve downward toward the black hole horizon meaning that the downward angle of each bit of energy along the string increases.

Figure 3 shows two examples of string evolution in vacuum, for a string with the initial condition (2.8) created at $z_{0}=0.02$ (above) and for a string with the initial condition (2.9) created at $z_{0}=0.01$ (below). We plot the time evolution of the downward angle of the point on the string above which a fraction $\epsilon$ of the string energy is found. That is, $\epsilon$ starts at 0 at the string endpoint and $\epsilon=0.5$ corresponds to the string midpoint, which by symmetry moves straight downward into the AdS bulk. For both strings in the figure, the initial downward angle of the string endpoint is zero. As a consequence of the string tension, during the early time dynamics each segment of the string changes its downward angle $\sigma$. For each segment of the string, though, after some time passes its downward angle no longer changes. That is, the string nullifies. The constant nonzero downward angle of the trajectory that the string endpoint follows after nullification, $\sigma_{0}$, is $1.8^{\circ}$ in the upper panel of figure 3 and $3.8^{\circ}$ in the lower panel.

The early time dynamics is particularly dramatic in the lower panel of figure 3 , where the endpoint of the string propagates almost horizontally for a time before relatively suddenly turning downwards with a nonzero angle that soon becomes constant. These strings, with the initial condition (2.9), describe a flow of energy that is initially collimated, with zero opening angle, before later, suddenly, acquiring a substantial opening angle. We shall describe this dynamics further in subsection 2.2. First, though, we shall provide a further description of the nullification process in vacuum and shall then illustrate that it works quite similarly in plasma - because nullification happens while the strings are still far 

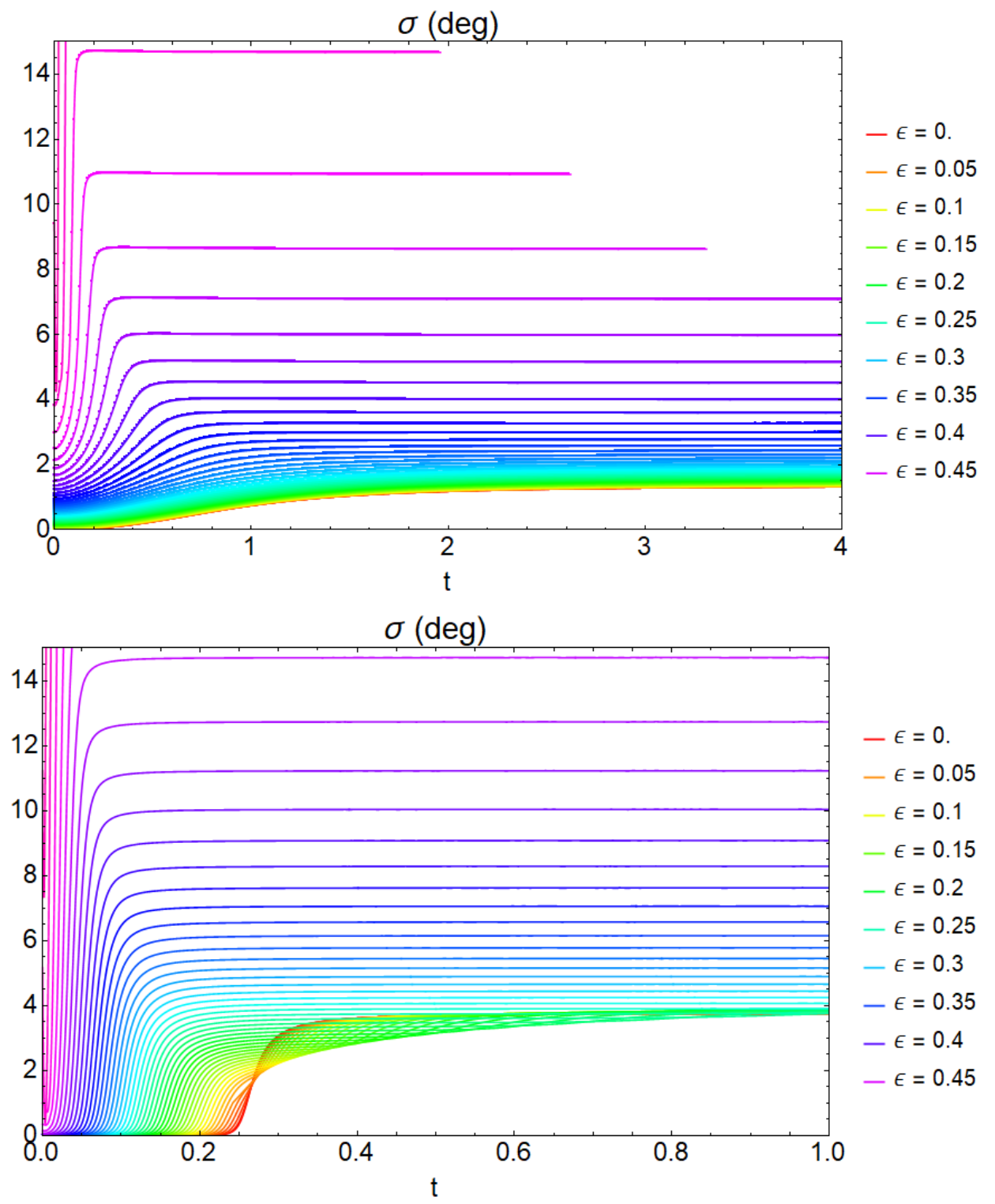

Figure 3. We depict the time evolution in vacuum of two strings whose endpoints initially move horizontally, with zero downward angle. The upper panel is for the initial condition (2.8) with $z_{0}=0.02$ and the lower panel is for the initial condition $(2.9)$ with $z_{0}=0.01$. Each curve shows the time evolution of the downward angle $\sigma$ (not to be confused with the worldsheet coordinate $\sigma_{w s}$ ) of the point on the string such that a fraction $\epsilon$ of the total energy of the string is found between that point and the string endpoint. Hence, $\epsilon=0$ corresponds to the string endpoint and $\epsilon=0.5$ corresponds to the string midpoint. The calculation is done entirely in vacuum. And, in the AdS vacuum null geodesics are trajectories with a constant downward angle $\sigma$. We see that each point on the string "nullifies": after some time, each bit of energy along the string moves along a null geodesic with some constant $\sigma$. After nullification, the string endpoints move along trajectories with nonzero downward angles. 
enough from the horizon that their dynamics is similar to that in vacuum. We shall see that even though the strings with initial conditions (2.9) have dramatic dynamics early on, after they nullify and become jet-like they look rather similar to the jet-like strings that form starting from the other initial conditions that we analyze.

\subsubsection{Nullification in vacuum}

In this subsection, we shall compute the nullification timescales and the resulting distribution of energy along the string as a function of the downward angle of the trajectory followed by a bit of string after nullification, for strings whose initial conditions are given by eqs. (2.8)-(2.13) that nullify in vacuum. The nullification process depends on the initial velocity profile, the energy of the half-string $E$ and the AdS depth $z_{0}$ of the point at which we initialize the string. We have analyzed nullification for the six classes of initial conditions presented above with varying values of $z_{0}$. Without loss of generality, in vacuum we can choose units of energy such that $E=1000$. For each string that we evolve, we compute the opening angle $\sigma_{0}$ with which the string endpoint is descending into the bulk after the string has nullified as well as the time $t_{\text {null }}$ it takes for the endpoint to reach this angle within accuracy of $10 \%$. In figures 4,5 and 6 we show these observables for our strings, and more.

In figure 4 (bottom) we see that if we choose initial conditions from one of our classes of initial conditions in which the initial downward angle of the string endpoint is zero then, after the initial phase of the string dynamics, when the string nullifies its endpoint is moving downward into the AdS bulk at a constant angle $\sigma_{0}$ that is well approximated by

$$
\sigma_{0} \sim \frac{c}{E z_{0}}
$$

for a profile-dependent constant $c$. Here, we have reinstated the $E$-dependence by dimensional analysis. (Strings that are initially closer to null, like those with initial conditions (2.10) and in particular (2.12), do not satisfy this relationship.) In ref. [143], it was shown analytically that in a limit in which $\sigma_{0} \rightarrow 0$ null strings as in figure 1 have an energy $E \propto \sigma_{0}^{-3 / 2}$. We now see that we can reproduce this limit by choosing a sequence of strings with zero initial downward angle and increasing $E$ as long as we initialize the strings at a $z_{0}$ that we choose to be $\propto E^{-1 / 3}$. The strings in this sequence will nullify with a $\sigma_{0} \propto E^{-2 / 3}$.

In figure 5 we plot the downward angle $\sigma$ of every point on each of the strings that we have studied, after nullification, rather than just focusing on the string endpoint. We find that for the strings with initial conditions in which the initial downward angle of the string endpoint was zero, after nullification the downward angle of a bit of string, scaled by the downward angle $\sigma_{0}$ of the endpoint of that string, takes on an approximate scaling form as a function of what fraction of the energy is found above that bit of string. This is equivalent to saying that the energy distribution along the string takes on an approximate scaling form after nullification. In section 3 when we use an ensemble of null strings to model an ensemble of QCD jets, we shall choose to distribute the energy density along these null strings according to the scaling form found in the lower panel of figure 5. Specifically, we shall take the form for $\sigma / \sigma_{0}$ as a function of $\epsilon$ found after nullification for strings that 

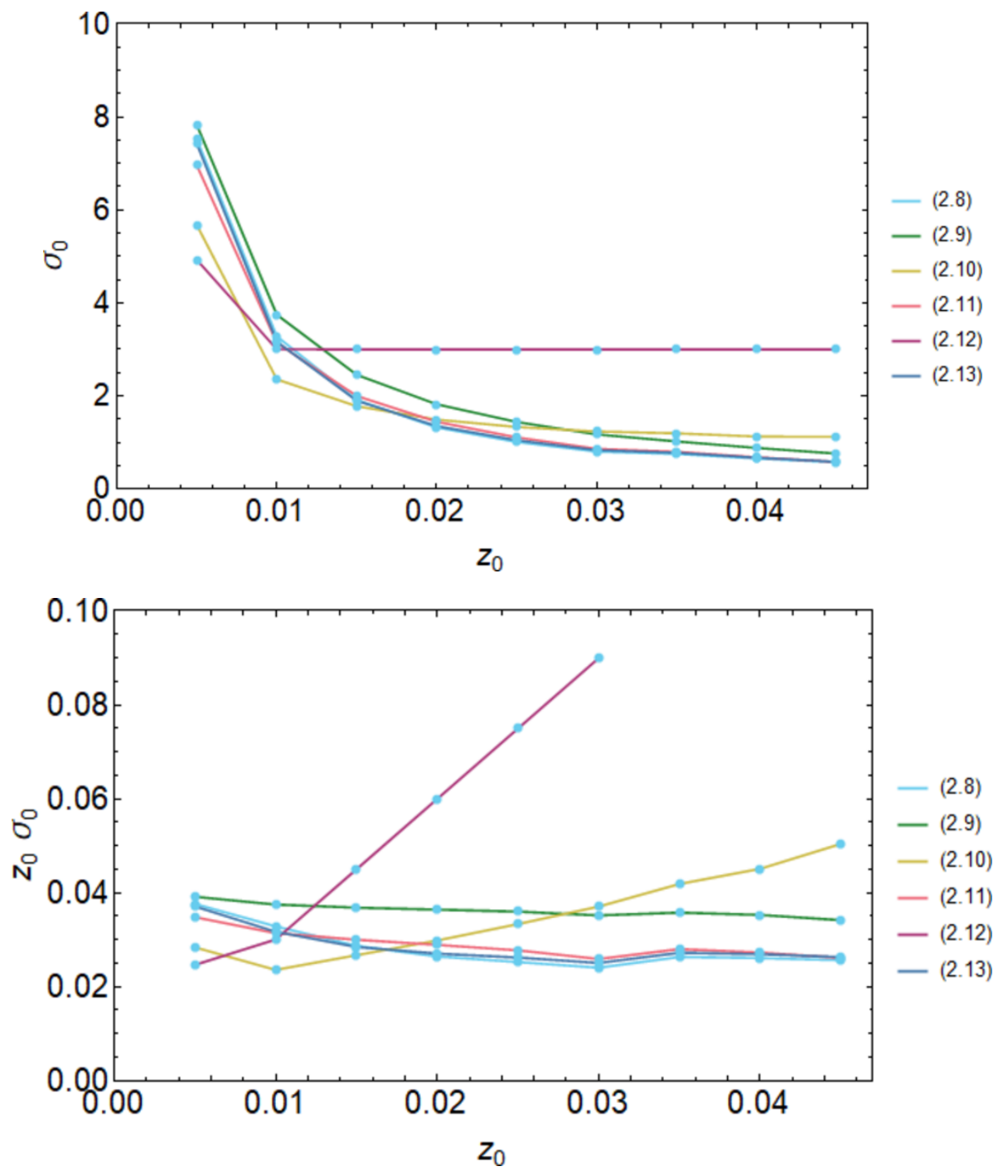

Figure 4. For each of the six classes of initial conditions in eqs. (2.8)-(2.13), we plot the downward angle $\sigma_{0}$ reached by the endpoint of the string after it nullifies, which is to say after the downward angle no longer changes. In the upper panel, we show how $\sigma_{0}$ varies as we change the AdS depth $z_{0}$ at which we initialize the string, while keeping the energy of the string fixed. We find that for strings with the initial conditions (2.8), (2.9), (2.11) and (2.13) in which the downward angle of the string endpoint is initially zero, to a good approximation the opening angle $\sigma_{0}$ reached by the string endpoint after nullification is given by $\sigma_{0} \sim \frac{1}{E z_{0}}$, with a prefactor $c$ that depends weakly on the initial string profile. For the initial conditions (2.10) and (2.12) in which the downward angle of the string endpoint is initially nonzero and in which the strings are closer to null from the beginning, this relationship is not satisfied.

start out at $z_{0}=0.005$ with the initial condition (2.8). This scaling form is the principal result of this section; it is the result from this section that we shall employ when we model an ensemble of jets in section 3 .

In the lower panel of figure 5 the scaling form that we find after nullification is compared to the result from ref. [141] (the black curve labelled 1511.07567), in which the near- 

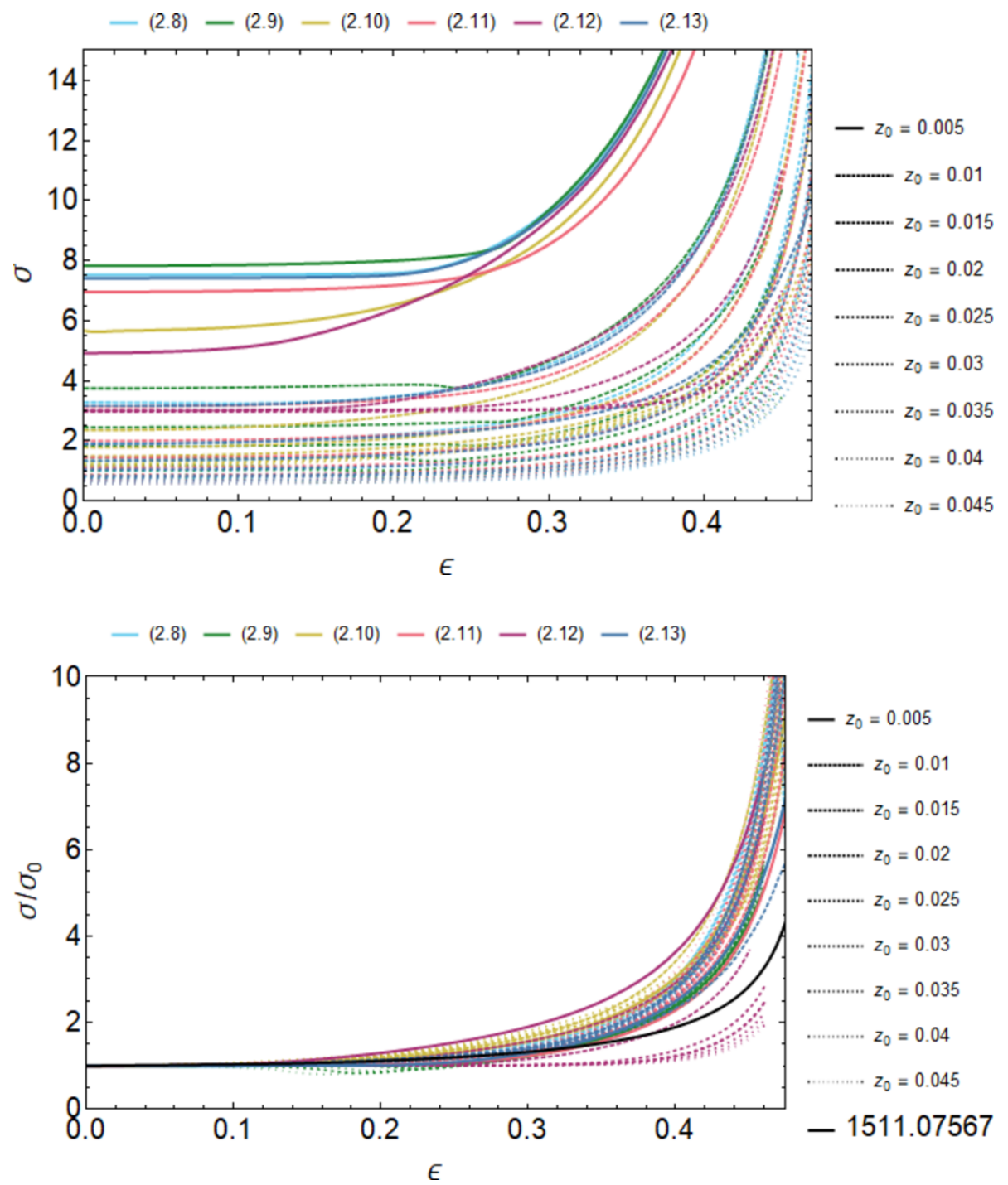

Figure 5. After nullification, the point on the string above which a fraction $\epsilon$ of the energy of the string is found moves along a null geodesic with some constant downward angle $\sigma$. In the upper panel, we plot this $\sigma$ as a function of $\epsilon$, after nullification, for strings with each of our six classes of initial conditions with varying values of $z_{0}$. Each color represents one of the initial conditions from eqs. (2.8)-(2.13) as shown at the top of the plot, and the degree of dashing indicates the value of $z_{0}$ used with that initial condition to produce the given curve. $\sigma_{0}$ is the value of $\sigma$ at $\epsilon=0$. In the lower panel, we rescale each of the many curves in the upper panel by its own $\sigma_{0}$. We find that for the strings with the initial conditions (2.8), (2.9), (2.11) and (2.13) in which the initial downward angle of the string endpoint was zero, after nullification the rescaled curves in the lower panel all take on a rather similar shape. This means that, for these initial conditions, the nullification dynamics rearranges the way that energy density is distributed along the string as a function of $\sigma / \sigma_{0}$ such that it reaches an approximate scaling form. The purple curves show that for initial conditions like (2.12) that are close to null from the beginning, because the energy density distribution along the string hardly changes it need not reach the scaling form. 
endpoint approximation for the energy density distribution as a function of $\sigma$, namely $e(\sigma) \propto \frac{1}{\sigma^{2} \sqrt{\sigma-\sigma_{0}}}$, was employed for the entire string. This provides a good approximation to the scaling form that we have found for $\epsilon \lesssim 1 / 4$, which is to say for the half of the energy of the string that is closer to its endpoint. Farther away from the endpoint, the near-endpoint approximation does not describe the scaling form that we have found.

Finally, in figure 6 we show the nullification times for every point on each of the strings that we have studied. There it can be seen that for those strings which start out far from null, with their endpoints moving horizontally, and subsequently nullify via the strongly coupled dynamics that we have focused on in this section do so after a nullification time that is around $(2-6) E z_{0}^{2}$, depending somewhat on the initial condition as well as on the position on the string.

\subsubsection{Nullification in plasma}

We shall now analyze the dynamics of strings with the initial conditions that we have introduced above in plasma, rather than in vacuum. When the strings are initially far above the black hole horizon, near the boundary, they are in a region of the spacetime where the metric is nearly the same as in vacuum. This means that strings which nullify quickly compared to the time it takes them to fall close to the black hole horizon will nullify via dynamics that is nearly the same as the dynamics in vacuum that we have analyzed above. We shall show in this subsection that, to good accuracy, this is indeed the case for strings with the initial conditions that we have chosen.

In vacuum, null geodesics are straight lines. This simplifies many computational aspects of studying nullification in vacuum compared to in plasma, because the angles of null geodesics stay fixed and the deviation from null is given by the deviation of the trajectory from a straight line and hence is easily assessed and quantified. As we discussed in section 1, in the AdS black hole spacetime dual to the plasma, null geodesics curve downward toward the horizon and a string gets represented by a congruence of null geodesics that loses energy to a wake in the plasma and ultimately thermalizes over a distance $x_{\text {therm }}$, the distance that the string endpoint travels before falling into the horizon. Because nullification happens relatively quickly compared to the thermalization time $x_{\text {therm }}$, we expect that it happens near the boundary where the spacetime is close to vacuum AdS. However, to test this we must have a way of assessing whether the string has nullified that can be applied in the regime where null geodesics curve downwards, namely in the regime that is not near the boundary. At any given time $t$ we construct a null geodesic that is tangent to the actual trajectory of the string endpoint (or for that matter to any chosen bit of string) and follow that null geodesic backwards/upwards all the way to the boundary. In the near-boundary region, this null geodesic is straight and has some downward angle. Once the string has nullified, its endpoint is following a null geodesic. This means that once the string has nullified, if we go to a later time and repeat the exercise of shooting a null geodesic that is tangent to the string endpoint trajectory back upwards we will find the same null geodesic with the same initial downward angle as we found at the earlier time. We define the angle $\sigma_{0}$ as this initial downward angle, defined from the near-boundary slope of the null geodesic that the string endpoint follows at late times. And we define the 

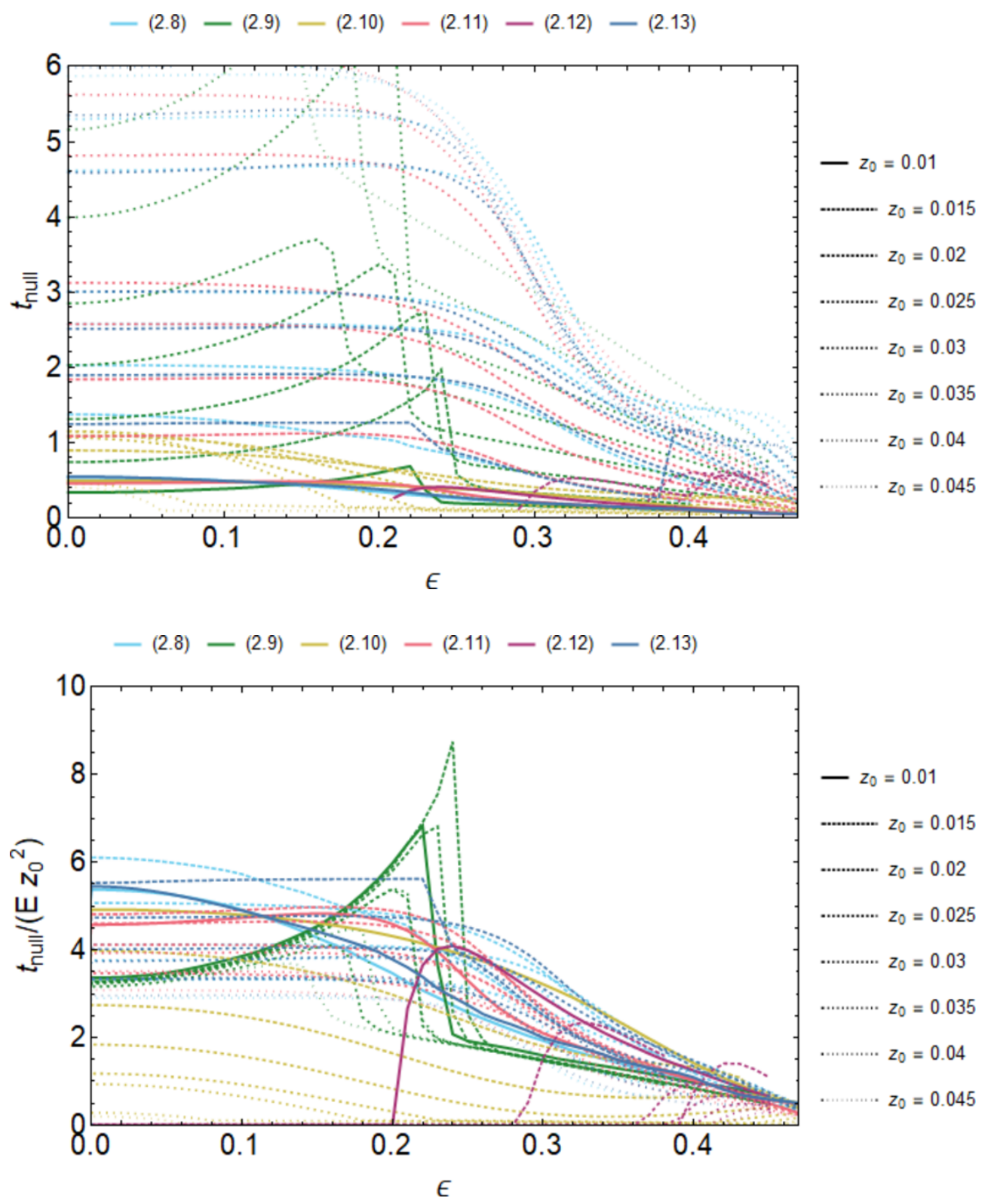

Figure 6. For a point on the string above which a fraction $\epsilon$ of the string energy is found, we define the nullification time $t_{\text {null }}$ as the time that the angle found in figure 5 is within $10 \%$ of its final value. In the upper panel we plot $t_{\text {null }}$ as a function of $\epsilon$ for the strings that we have analyzed. In the lower panel, we plot $t_{\text {null }} /\left(E z_{0}^{2}\right)$, and find that the nullification time is roughly proportional to $z_{0}^{2}$ for the strings whose endpoints had an initial downward angle of zero. Each color represents one of the initial conditions from eqs. 2.8)-(2.13 as shown at the top of the plot, and the degree of dashing indicates the value of $z_{0}$ used with that initial condition to produce the given curve. Those strings which we initialized with initial conditions (2.10) with their endpoints moving with a nonzero downward angle are closer to null from the beginning, meaning that it is no surprise that they nullify faster. 

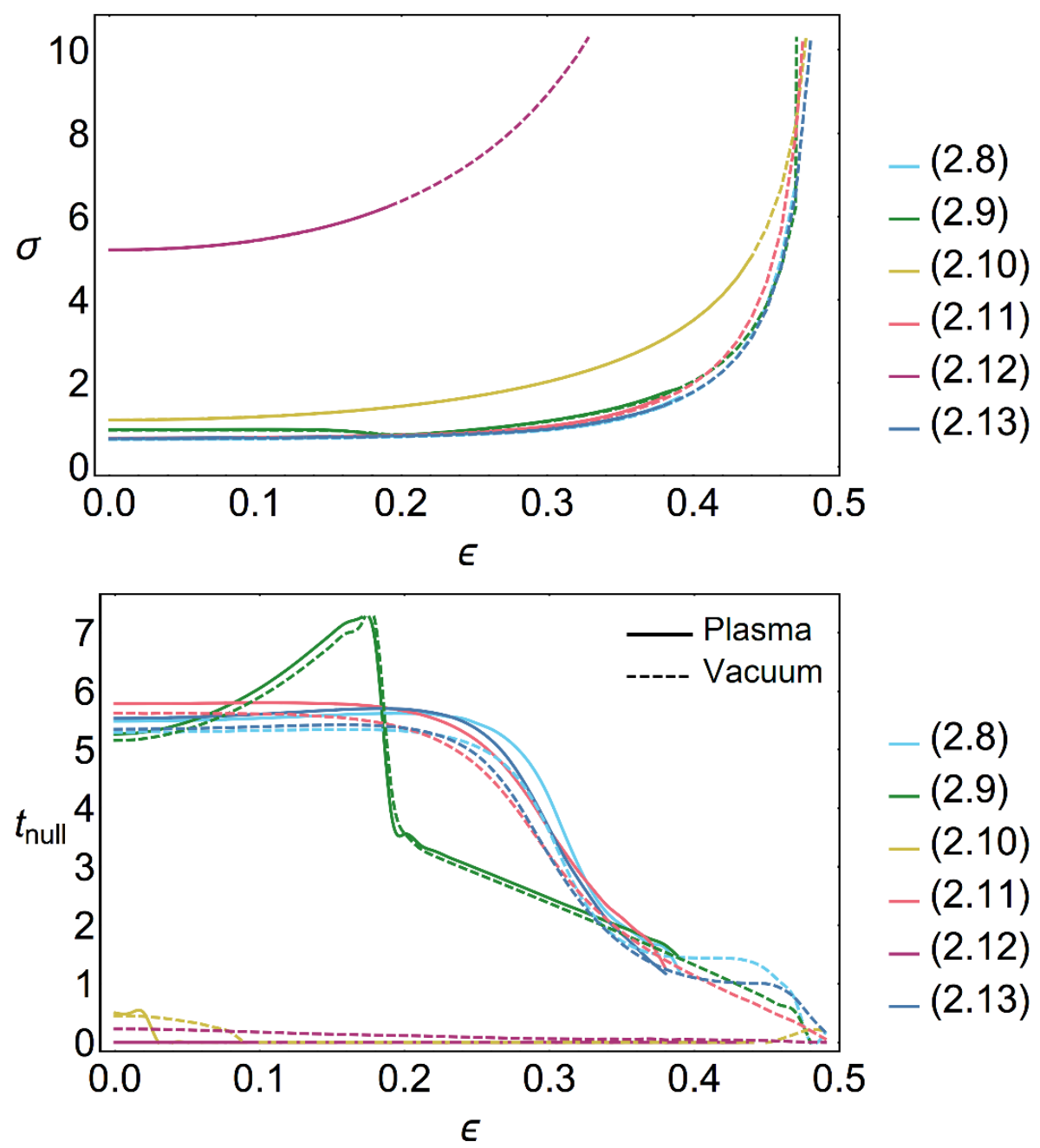

Figure 7. We compare the downward angles of the string endpoints after nullification $\sigma$ defined as described in the text (top panel) and the nullification times (bottom panel) for strings that are produced and then nullify in plasma (solid curves) to those that are produced and then nullify in vacuum (dashed curves) as in the previous subsection. All strings were produced at $z_{0}=0.04$ except for those with initial conditions (2.12), which were produced at $z_{0}=0.005$.

nullification time $t_{\text {null }}$ as the time after which this initial downward angle changes by less than $10 \%$. Defined in this way, both $\sigma_{0}$ and $t_{\text {null }}$ can be compared directly to their values in vacuum, allowing for the quantitative comparison between the nullification dynamics in plasma to that in vacuum depicted in figure 7 . We see that the nullification dynamics is similar indeed, confirming that nullification happens quickly enough that, in plasma, it happens in the near-boundary regime where the spacetime is very similar to vacuum AdS.

We conclude that the strings that we investigate nullify far above the horizon, near the boundary. This means that the expression (1.2) provides a reasonable approximation to the relationship between the distance that they travel between nullification and thermalization, $x_{\text {therm }}$, and their initial downward angle after nullification $\sigma_{0}$, defined as described above. It also completes the justification for how we shall model jets in section 3: we shall use null 
strings as in figure 1 with a specified initial downward angle $\sigma_{0}$ and with energy density distributed along the string according to the scaling form that we have found via our analysis in subsection 2.1.1 of the dynamics of how strings nullify in vacuum.

\subsection{Strings with most of their energy at their endpoint}

Before turning to modeling jets, we close this section with a further look at the nullification of strings with the initial condition (2.9), noting that this is close to a particular string initial condition from ref. [139] in which all of the energy and momentum of the string is initially localized at its endpoint, and hence travels initially in the same direction. We have seen that the dynamics of these strings before nullification is dramatic. However, these strings nullify like the others and have the benefit that they allow a partial analytic treatment, one that will allow us to confirm (in this special case) some of the scaling expressions that we found above more generally, but numerically. It has been shown in the literature that if we choose initial conditions in which all of the string energy is localized at its endpoint with the string endpoint initially moving parallel to the boundary at some $z_{0} \ll 1 /(\pi T)$, with zero downward angle, then the endpoint loses energy according to $[139,140]$

$$
\frac{d E}{d x}=-\frac{\sqrt{\lambda}}{2 \pi} \frac{1}{z_{0}^{2}} .
$$

The endpoint will follow a straight line (i.e. a null geodesic) at constant $z_{0}$ until all its energy has been depleted, which happens after a distance:

$$
x_{\text {snap }}=\frac{2 \pi}{\sqrt{\lambda}} E z_{0}^{2} .
$$

At that point the endpoint cannot continue further along its initial null geodesic and must change direction (a "snapback"), as can clearly be seen in the lower panel of figure 3. (With $E=1000, \lambda=5.5$ and $z_{0}=0.01$, the expression (2.19) yields $x_{\text {snap }}=0.27$, in agreement with the behavior seen in that figure.) This means that at this point in time the string is clearly different from a null string and hence we find that for this particular type of strings $t_{\text {null }} \geq(2 \pi / \sqrt{\lambda}) E z_{0}^{2}$, consistent with the approximate scaling that we found more generally, but numerically, above.

After this snapback, nullification occurs: the string endpoint finds itself moving along a new null geodesic with some nonzero downward angle $\sigma_{0}$ that does not change further. In order for the endpoint to continue on a null geodesic without another snapback we find a condition on the initial endpoint energy $E_{0}$, its starting position $z_{0}$, and $\sigma_{0}$, the opening angle after nullification, that is given by

$$
E_{0} \geq \int_{z_{0}}^{\infty} d z \frac{d E}{d z}=-\int_{z_{0}}^{\infty} d z \frac{\sqrt{\lambda}}{2 \pi} \frac{1}{z^{2} \sqrt{1-f / R^{2}}}
$$

where $R=-f \sqrt{\dot{x}^{2}+\dot{z}^{2}} / \dot{x}=1 / \cos \left(\sigma_{0}\right)$, which is to say by

$$
E_{0} \geq \int_{z_{0}}^{\infty} d z \frac{1}{z^{2} \sin \left(\sigma_{0}\right)}=\frac{1}{\sin \left(\sigma_{0}\right) z_{0}}
$$

which is again consistent with the more general scaling that we found numerically above. 


\section{A model for an ensemble of jets in heavy ion collisions}

We are now ready to construct the ensemble of null strings in the dual description of $\mathcal{N}=4$ SYM theory that we shall use as a model for an ensemble of jets in heavy ion collisions. The first step is to understand the relationship between the energy density distributed along an individual null string and the shape of the individual jet that this string represents. Here by shape we mean the distribution of $P_{\text {out }}$, the outward-directed flux of power at infinity, as a function of the angle $r$ measured from the center of the jet. We use the result from ref. [141]:

$$
\frac{d P_{\mathrm{out}}}{d \cos r}=\frac{1}{2} \int_{\sigma_{0}} d \sigma \frac{e(\sigma)}{\gamma(\sigma)^{2}[1-v(\sigma) \cos r]^{3}},
$$

where as in figure 1 we have parametrized the null string worldsheet by $\sigma$, the initial downward angle of a blue null geodesic along which a bit of energy travels, where $e(\sigma)$ is the energy density along the string as a function of $\sigma$ with $E_{\text {final }}=\int_{\sigma_{0}} d \sigma e(\sigma)$, where $\gamma(\sigma) \equiv\left(1-v(\sigma)^{2}\right)^{-1 / 2}$, and where $v(\sigma)=\cos \sigma$ for a null geodesic. This formula is relevant for a null string propagating through a finite droplet of QGP that emerges from that droplet and then propagates onward to infinity in vacuum. The domain of integration is over the angles $\sigma$ that label those blue null geodesics that do not fall into the black hole. We will choose an ensemble of strings with differing values of the initial downward angle of the string endpoint, $\sigma_{0}$. We shall specify the probability distribution for $\sigma_{0}$ and for the initial energy of the string below. For an individual string in the ensemble with some particular value of $\sigma_{0}$, we choose $e(\sigma)$ to be given by the approximate scaling form that we found in section 2.1.1 for the distribution of energy along the string after nullification for strings that were initially not null, whose endpoints initially had no downward angle. The approximate scaling form found after nullification is illustrated in the lower panel of figure 5 , where what is plotted is $\sigma / \sigma_{0}$ as a function of $\epsilon$, for points on the string above which a fraction $\epsilon$ of the total energy of the string. Specifically, we use the curve from figure 5 for strings that start out at $z_{0}=0.005$ with the initial condition (2.8). For a string of total energy $E$ whose downward endpoint angle at nullification is given by $\sigma_{0}$, we denote the curve in the lower panel of figure 5 by $\sigma(\epsilon)$ and express the jet shape $P_{\text {out }}(r)$ as a function of the angular coordinate $r$ away from the jet axis as

$$
P_{\text {out }}(r)=\int d \epsilon \frac{E \sin r}{\gamma(\sigma(\epsilon))^{2}[1-v(\sigma(\epsilon)) \cos r]^{3}} .
$$

This relation allows us to compute the shape of individual model jets in the $\mathcal{N}=4 \mathrm{SYM}$ gauge theory corresponding to null strings with a specified initial downward angle $\sigma_{0}$.

Next, we must specify the distribution of the initial energy $E$ and downward angle $\sigma_{0}$ of the strings in the ensemble that we shall use to model an ensemble of jets. We shall then be able to compute the mean jet shape in the ensemble in vacuum. We will then construct an ensemble of dijets, using the probability distribution of the dijet asymmetry measured in proton-proton collisions. We will also need a model for the evolution of the plasma and a distribution for the starting points and directions of the jets in the transverse plane. After sending our jets through an expanding cooling droplet of strongly coupled $\mathcal{N}=4$ 
SYM plasma, we shall investigate how the mean jet shape and the distribution of the dijet asymmetry are modified by passage through the plasma.

As in ref. [145], we shall utilize perturbative QCD calculations of the probability distribution for a useful measure of the opening angle of a jet in QCD defined by

$$
C_{1}^{(1)} \equiv \sum_{i, j} z_{i} z_{j} \frac{\theta_{i j}}{R},
$$

where the sum is over all pairs of hadrons in the jet, $\theta_{i j}$ is the angle between hadrons $i$ and $j$, and $z_{i}$ is the momentum fraction of hadron $i$. We shall consider jets reconstructed with the anti- $k_{t}$ algorithm [154] with reconstruction parameter $R=0.3$, as in the CMS data that we shall compare our results to below. We shall (quite arbitrarily) take the quark and gluon fractions each to be 0.5 in the formulae for the $C_{1}^{(1)}$ distribution calculated in perturbative QCD in ref. [155]. The opening angle of a holographic jet is proportional to the downward angle of the string endpoint $\sigma_{0}$ [143], but we have no direct analogue of $C_{1}^{(1)}$ since the holographic calculation does not have hadrons meaning that we cannot calculate eq. (3.3) explicitly. Therefore, as in ref. [145] we shall take

$$
C_{1}^{(1)}=a \sigma_{0}
$$

introducing a free parameter $a$ in our model. This allows us to translate the perturbative QCD calculations for the distribution of $C_{1}^{(1)}$ into a probability distribution for the initial downward angle $\sigma_{0}$ of the strings in our ensemble. Note that this probability distribution depends on the initial jet energy $E$. We complete the specification of our ensemble of strings by choosing a distribution of initial jet energies which falls as $E^{-6}$.

The differential jet shape for an individual jet is the power $P_{\text {out }}(r)$ as a function of the angle $r$ from the jet axis, as given in eq. (3.2). The (normalized) mean jet shape is the average of the individual jet shapes over the ensemble. We plot our results for the mean jet shape in figure 8 , binning them in bins of width $\Delta r=0.05$ for consistency with the CMS data, which we also show in the figure. We find that upon making a suitable choice for the free parameter in the model $a$ we obtain a rather good description of the mean jet shape measured in proton-proton collisions! The result shown in figure 8 has the best fit value $a=2$ shown in red, which is in reasonable agreement with the crude estimate of $a \sim 1.7$ given in [145] for smooth jets, as well as a band of predictions corresponding to varying $a$ from 1.8 to 2.5. We find it pleasing, and perhaps even remarkable, that even though we picked the initial energy distribution along the null strings that we are using to model jets from a strongly coupled calculation of the dynamics of strings as they nullify that is quite different from the dynamics of jets in QCD, the mean jet shape that we obtain agrees so nicely with measurements made in proton-proton collisions.

To compute the modification to the mean jet shape in our ensemble caused by the passage of the jets through the strongly coupled plasma, we need a model for the dynamics of the droplet of plasma. We assume boost invariant longitudinal expansion and initialize the droplet of plasma at a proper time $\tau=1 \mathrm{fm} / \mathrm{c}$ after the collision. As in ref. [145], we make the overly simplified assumption that our null strings are produced at the same time 


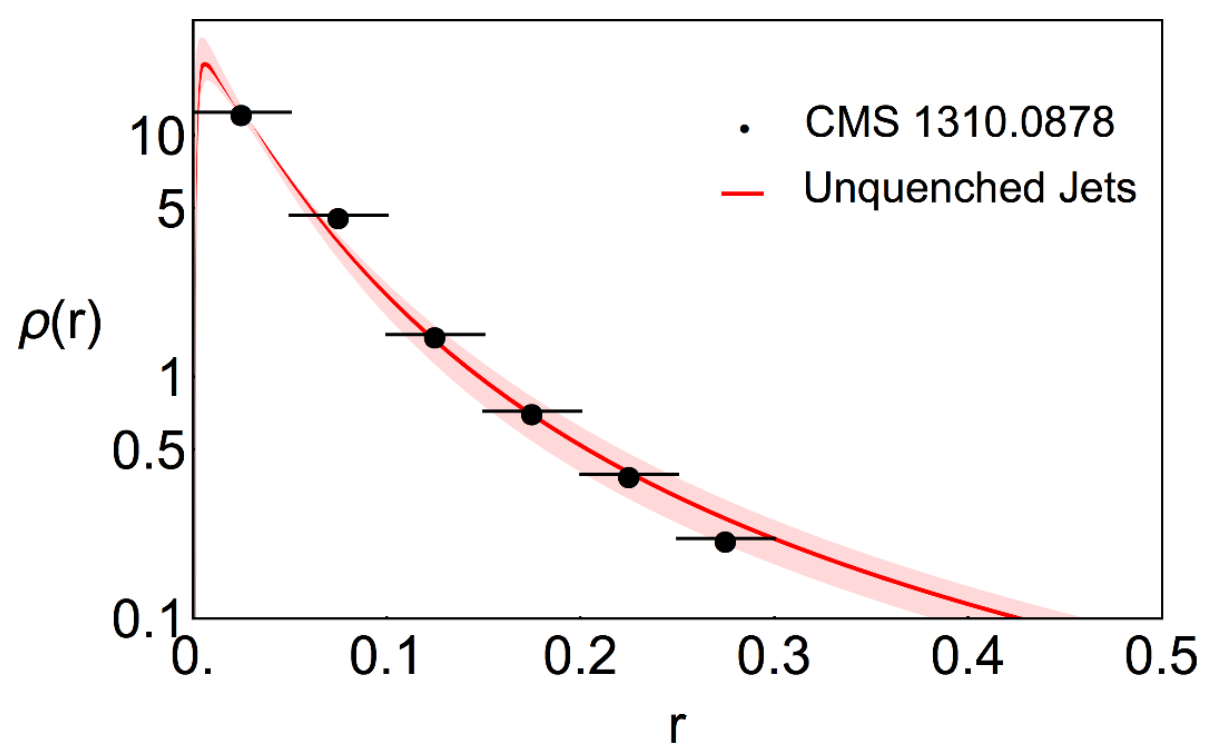

Figure 8. Mean jet shape in vacuum as a function of $r$, the angle in $(\eta, \phi)$-space from the center of the jet, computed from the ensemble of null strings described in the text compared to CMS measurements of the mean jet shape for jets with energy above $100 \mathrm{GeV}$ reconstructed with anti- $k_{t}$ reconstruction parameter $R=0.3$ in proton-proton collisions with $\sqrt{s_{\mathrm{NN}}}=2.76 \mathrm{TeV}$ at the LHC from ref. [33], shown as black symbols. The pink band shows the results obtained from our ensemble of strings for a range in the free parameter given by $a=1.8-2.5$, with $a=2$ shown in dark red. Although the effects of doing so are negligible here, the experimental data and the results of our calculations have been smeared in order to take into account the CMS jet energy resolution, as described in ref. [33] and later in the text.

that the hydrodynamic plasma is initialized, thus completely neglecting the possibility of energy loss before the plasma hydrodynamizes. Again as in ref. [145], we also make the overly simplified assumption that all quenching stops and the strings propagate as in vacuum after the droplet of plasma has cooled below $T=175 \mathrm{MeV}$. The droplet of $\mathcal{N}=4$ SYM plasma and its evolution are encoded, via the AdS-CFT correspondence, in changes to the 5-dimensional metric in AdS space. An expanding and cooling droplet of plasma in the field theory corresponds to a black hole in 5-dimensional AdS space whose horizon is expanding in the spatial directions while shrinking "downward", away from the AdS boundary, in the $z$-direction. As in ref. [145], we take a simple blast-wave profile to model the temperature evolution in the transverse plane and assume boost-invariant longitudinal expansion, choosing

$$
T\left(\tau, \vec{x}_{\perp}\right)=b\left[\frac{d N_{\mathrm{ch}}}{d y} \frac{1}{N_{\text {part }}} \frac{\rho_{\text {part }}\left(\vec{x}_{\perp} / r_{\mathrm{bl}}(\tau)\right)}{\tau r_{\mathrm{bl}}(\tau)^{2}}\right]^{1 / 3} .
$$

Here, $\tau \equiv \sqrt{t^{2}-z^{2}}$ is the proper time, $\rho_{\text {part }}\left(\vec{x}_{\perp}\right)$ is the participant density in the transverse plane as given by an optical Glauber model, and $r_{\mathrm{bl}}(\tau) \equiv \sqrt{1+\left(v_{T} \tau / R\right)^{2}}$ with $v_{T}=0.6$ and $R=6.7 \mathrm{fm}$. We consider $2.76 \mathrm{TeV} \mathrm{Pb-Pb}$ collisions at mid-rapidity and $0-10 \%$ centrality at the LHC, and based upon averaging the results for $0-5 \%$ and $5-10 \%$ centrality from 


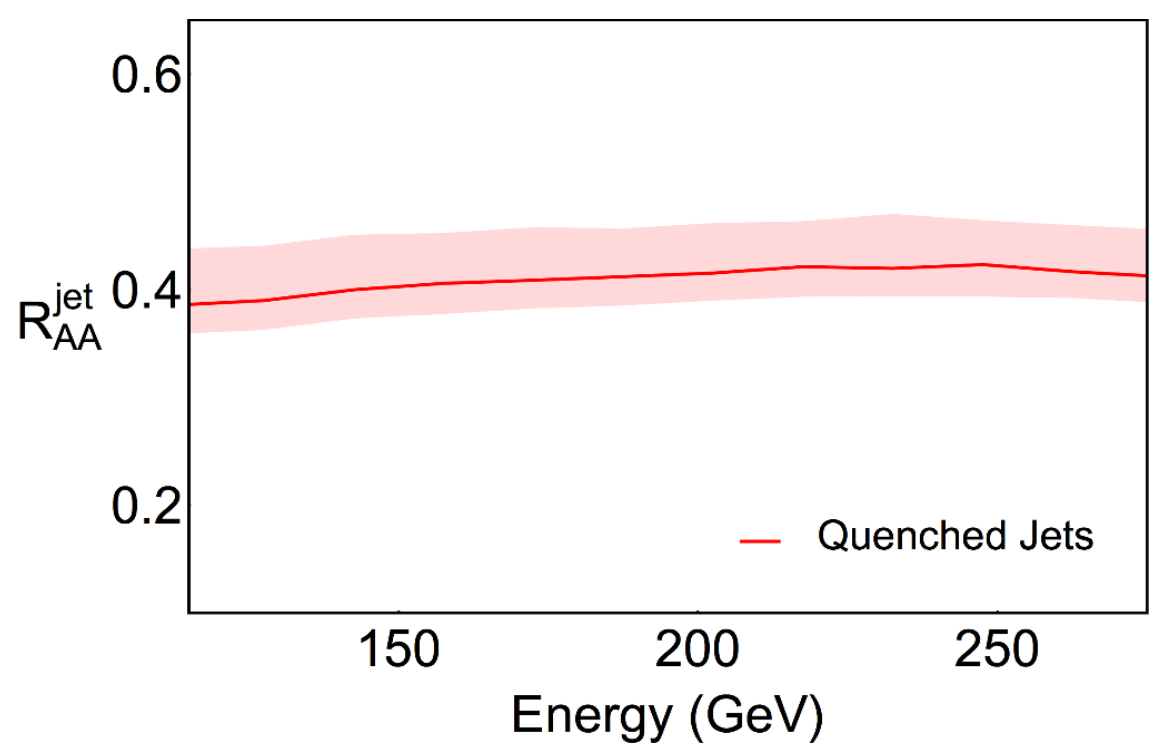

Figure 9. Jet suppression $R_{\mathrm{AA}}^{\text {jet }}$, the ratio of the number of jets with a given energy in our ensemble after the jets have been quenched via passage through the expanding cooling droplet of plasma to the number of jets with the same energy in the initial ensemble, before quenching. As in figure 8, the pink band shows the results obtained from our ensemble of strings for a range in the parameter $a$ given by $a=1.8-2.5$, with $a=2$ shown in dark red. We have chosen the value of $b$, the second free parameter in the model, defined in the text, to be $b=0.21$ so as to obtain a jet suppression that is comparable to experimental measurements of jet $R_{\mathrm{AA}}$, for example those in refs. [31, 38, 46].

ref. [156] we take the number of participants to be $N_{\text {part }} \simeq 356$ and based upon summing the results for pions, kaons and protons in ref. [157] we take $d N_{\mathrm{ch}} / d y \simeq 1599$. $b$ is the second free parameter in our model; we shall use it to parameterize differences in the number of degrees of freedom between $\mathcal{N}=4 \mathrm{SYM}$ and QCD, meaning that we should model a QCD plasma with temperature $T$ by an $\mathcal{N}=4$ SYM plasma with some lower temperature. The constant $b$ is a measure of the multiplicity per entropy, and for a QCD plasma is $b \approx 0.78$ [145]. Since we are modeling this plasma with an $\mathcal{N}=4 \mathrm{SYM}$ plasma, in our model we must scale the temperature that we use down by choosing a value of $b$ that is substantially smaller than this [145].

In this work, we consider an ensemble of $\approx 150,000$ jets which sample distributions in jet opening angle, energy, and the starting position and direction of the jets within the droplet of plasma. We take the initial position of the quark-antiquark pair in the transverse plane to be distributed according to a binary scaling distribution proportional to $\rho_{\text {part }}\left(\vec{x}_{\perp}\right)^{2}$ with their directions randomly distributed in the transverse plane. We have described our choice for the initial distribution of the energy and opening angle, and hence downward angle for the null string endpoint, above. For the analysis of the dijet asymmetry, we additionally sample the initial dijet asymmetry distribution, which increases the size of our ensemble by a factor of roughly 30 .

After we send our ensemble of strings through the expanding cooling droplet of plasma, we recompute the energies and opening angles (given by $a$ times the downward angle of the 
string endpoint after it emerges from the droplet of plasma) of each string in the ensemble. In the next section, we shall compute the modification to various observables from the ensemble after passage through the plasma. The simplest observable to calculate is the ratio of the number of jets with a specified $E$ in the ensemble after quenching to that number in the ensemble before quenching, a ratio that is the analogue in our model of $R_{\mathrm{AA}}^{\mathrm{jet}}$. In figure 9 we show that if we choose $b=0.21$ we obtain a value for this ratio that is comparable to the measured value of $R_{\mathrm{AA}}^{\mathrm{jet}}$ [31]. This value of $b$ is qualitatively consistent with what has been found in other models. For example, in the hybrid strong/weak coupling model for jet quenching, fitting the single model parameter in that model to experimental measurements of $R_{\mathrm{AA}}^{\mathrm{jet}}$ yields the conclusion that the thermalization length $x_{\text {therm }}$ for jets is about 3-4 times longer in QCD plasma with temperature $T$ than in strongly coupled $\mathcal{N}=4$ SYM plasma with the same temperature $[147,148]$.

The blast wave temperature evolution is of course much simpler than the true hydrodynamic evolution of the strongly-coupled plasma in heavy ion collisions. In addition, the use of the metric (2.1) with a $T$ that varies in space and time has the downside that it neglects flow, viscosity, and gradients in the plasma: (2.1) itself is the metric corresponding to a constant-temperature plasma and when we insert a $T$ that varies in space and time into it what we obtain is not a solution to Einstein's equations for a plasma whose temperature varies in space and time, meaning that it does not describe a solution to hydrodynamics: as noted, it neglects flow, viscosity and gradients. We have done brief and preliminary investigations where we have chosen more realistic hydrodynamic backgrounds. First, we have tried taking the temperature evolution from the viscous hydrodynamic simulation of a heavy ion collision in ref. [158] and used the metric (2.1) for this $T$ that varies in space and time. Second, we have tried taking both the temperature and fluid velocity from ref. [158] and implementing higher order gradient corrections to the metric to include the effects of flow and viscosity, as well as gradients in the fluid as in ref. [159]. From this brief study, it appears that the alternative temperature profiles have only small quantitative effects on the energy loss, while the presence of flow and gradients may have somewhat larger effects but effects that are still only quantitative, not qualitative. Because of the computational complexity of such calculations, we postpone the inclusion of a full hydrodynamic background including fluid velocity, viscosity and gradients in the analysis of the full ensemble of jets to future works. In our limited study, including flow in the plasma profile appears to decrease the energy loss. Improving upon our oversimplified treatment (aka neglect) of energy loss before hydrodynamization and after hadronization would increase the energy loss; we leave these investigations to future work also.

Before we turn to our results, we note that we shall be comparing our results for how various observables are modified by passage through the plasma to CMS measurements of jets in $\mathrm{PbPb}$ collisions from which the effects of the jet energy resolution of the detector have not been unfolded. This means that, as described in refs. [27, 33], the appropriate baseline against which to compare these measurements is not data from proton-proton collisions per se, because the jet energy resolution of the CMS detector differs in $\mathrm{PbPb}$ and proton-proton collisions. What we need to do, therefore, is to take as inputs to our calculations distributions appropriate for unsmeared proton-proton collisions (we shall use PYTHIA 
simulations thereof), calculate the modifications to observables after we run our ensemble of jets through a droplet of plasma, and then before comparing to measured data we must smear the jet energy in our initial (proton-proton) ensemble and in our final ensemble after quenching, in both cases using the Gaussian smearing functions for $0-10 \%$ centrality $\mathrm{PbPb}$ collisions provided by the CMS collaboration in ref. [28]. In figures 8 and 9 and in the following section, we can then compare to CMS measurements in $\mathrm{PbPb}$ collisions that have not been unfolded, and to the pp baseline against which CMS compares these measurements, namely simulated proton-proton jets smeared to take into account the difference in the jet energy resolution of the $\mathrm{CMS}$ detector in $\mathrm{PbPb}$ and proton-proton collisions.

\section{Results, conclusions and outlook}

In previous sections, we have described our construction of an ensemble of null strings in the dual gravitational description of $\mathcal{N}=4$ SYM theory that we shall use as a model for an ensemble of jets. Using null strings means that we are automatically describing flows of energy whose opening angles do not change, in vacuum, making them natural as models for high energy jets in QCD whose jet mass and jet energy do not change, in vacuum. Building an ensemble in such a way that it can serve as a model for an ensemble of jets produced in proton-proton collisions required us to use key further inputs from several different directions. First, we chose the probability distribution for the energy and opening angle of the jets represented by the strings in our ensemble from perturbative QCD calculations of these quantities in proton-proton collisions. This alone is not enough, however, as we must specify the distribution of energy density along our null strings. We have done so in a way that incorporates a striking regularity of the dynamics of strings in AdS that we identified in section 2. We found that a large class of strings that are initially not null, and in particular that have a vanishing initial opening angle, evolve to become null, and as they do the energy density distributed along them takes on a particular scaling form, when scaled relative to the downward angle of the string endpoint, which is proportional to the opening angle of the jet that the string models. In section 3 we have seen that if we choose the probability distribution for the opening angles of the jets in our ensemble according to the results of a perturbative QCD calculation, and then distribute the energy density along the string that represents each jet according to the scaling form that we identified from our analysis of string dynamics, we obtain an ensemble of jets with a mean jet shape that is in excellent agreement with that measured in proton-proton collisions, see figure 8 , upon fixing $a$, the first of two free parameters in our model.

One way of thinking about the way that we have chosen our ensemble of strings is that we have only included a subset of all possible null strings, the subset whose energy is distributed along them such that they can be obtained by starting with non-null strings whose initial opening angle vanishes initially and letting these non-null strings evolve. The string dynamics turns these initially non-null strings into jet-like, null, strings with a particular form for their energy density distribution. Like any null string, these null strings can be created by initializing them that way. But, this subset of null strings can also be created by starting with non-null initial conditions and letting the string evolve and 
nullify. Although the property of the string dynamics that we are employing is striking, we have no first-principles argument for why we should choose this subset of null strings. Similarly, we do not know whether the only strings that can reasonably be used as models for jets are strings that are null from the beginning. We leave to future work constructing the bulk-to-boundary propagator for non-null strings which nullify, computing the gauge theory stress-energy tensor at early times, and investigating whether there are some initially non-null strings whose opening angle is reasonably constant at all times, including before nullification. With such a computation of the gauge theory stress-energy tensor at early times in hand, further investigations would also be possible, including trying to design initially non-null strings that have a reasonably constant opening angle at early times and that at the same time have (fractal) substructure, as QCD jets do. For the present, we have an ensemble of strings that model an ensemble of jets in vacuum with a mean jet shape as shown in figure 8 .

Next, we send this ensemble of strings through the simplified model for the expanding, cooling droplet of plasma that we have described in section 3. There are many ways in which our treatment of the medium that our ensemble of strings sees could be improved in future work. As noted in section 3, we have neglected any interactions between the strings and the medium before $\tau=1 \mathrm{fm} / c$ and after the time when the plasma cools below $T=175 \mathrm{MeV}$. Both these oversimplifications can be revisited in future work. Again as described in section 3, we have used a blast wave model for the dynamics of the expansion and cooling of the droplet of plasma; this can be revisited too. At the same time in future when a full relativistic viscous hydrodynamic treatment of the plasma is used instead of a blast wave model, the AdS black hole metric should be augmented to include the effects of shear viscosity and of fluid gradients. For the present, we have the blast wave model for the expanding, cooling droplet of plasma, as described in section 3. There, we have fixed the parameter $b$ (which is the proportionality constant between the temperature of the QCD plasma and the temperature of the $\mathcal{N}=4 \mathrm{SYM}$ plasma - with its greater number of degrees of freedom - that we are using to model the QCD plasma). The value of $b$ that we find corresponds to choosing the $\mathcal{N}=4 \mathrm{SYM}$ plasma to be between 3 and 4 times cooler than the QCD plasma that we are modeling, consistent with other estimates made in quite different ways $[147,148]$.

After sending our ensemble of jets through the droplet of expanding, cooling plasma, we calculate the new mean jet shape in the ensemble, after quenching. Note that, as in any experimental analysis, we impose a cut on the jet energy. In order to compare our results for the modification of the mean jet shape to the experimental measurements in ref. [33], we impose the cut $p_{T}^{\text {jet }}>100 \mathrm{GeV}$, meaning that any jet whose transverse momentum drops below $100 \mathrm{GeV}$ upon propagation through the plasma is removed from the ensemble. This means that even though every jet in the ensemble gets wider as it propagates through the plasma, because jets that are initially wider lose more energy and hence are more likely to drop below $100 \mathrm{GeV}$ the mean opening angle of the jets in the ensemble can decrease [145]. Indeed, when we plot the ratio of the mean jet shape of the jets in our ensemble after quenching to that in our ensemble before quenching in figure 10 we see that quenching makes the mean jet shape get narrower in our calculation. In figure 10 we compare the 


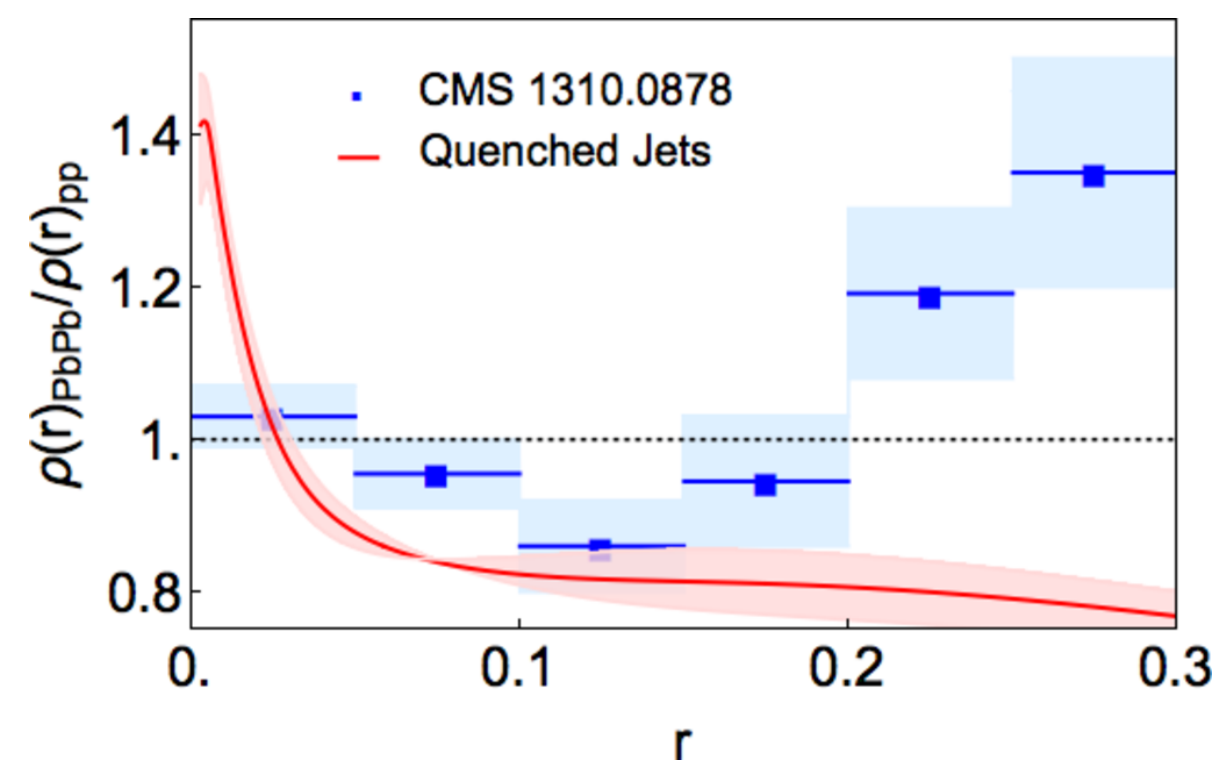

Figure 10. The ratio of the mean shape of the jets in our ensemble after they have been quenched by their passage through the expanding cooling droplet of plasma, as described in section 3 , to their mean shape before quenching, shown in figure 8. As in figures 8 and 9, the pink band shows our results for $a=1.8-2.5$ with $a=2$ shown in dark red. The CMS measurement [33] of the modification of the mean jet shape, namely the ratio of its measured value for jets with energy above $100 \mathrm{GeV}$ reconstructed with anti- $k_{t} R=0.3$ in $0-10 \%$ central $\mathrm{PbPb}$ collisions with $\sqrt{s_{N N}}=2.76 \mathrm{TeV}$ to that in proton-proton collisions, as in figure 8 , is shown as the blue symbols. The discrepancy between our calculation and the experimental measurements at large angles $r$ relative to the center of the jet is expected, since we do not include any analogue of the particles originating from the wake that the jet leaves behind in the plasma, some of which must necessarily be reconstructed as a part of the jet in any experimental analysis even after background subtraction [149].

results of our calculation to the CMS data of ref. [33], finding rough qualitative agreement at small $r$, namely close to the core of the jet, where we see that the ratio plotted in the figure drops below one in our calculation and in data. This confirms that even though every jet in the ensemble gets wider as it propagates through the plasma, the mean jet shape of the jets in the ensemble with $p_{T}>100 \mathrm{GeV}$ gets narrower. At larger $r$, our model does not include the soft particles coming from the wake in the plasma - which carry the momentum lost by the jet and which therefore must contribute to the reconstructed jet [149]. Including these effects increases the number of soft particles at all angles in the jet cone, which pushes the ratio plotted in figure 10 significantly upwards at larger $r$ [149].

A by now classic signature of the modification of jets in heavy ion collisions due to their passage through the strongly coupled plasma is a significant enhancement in the dijet asymmetry. In events in which at least two jets are reconstructed, the dijet asymmetry is defined as $A_{J} \equiv\left(p_{T, 1}-p_{T, 2}\right) /\left(p_{T, 1}+p_{T, 2}\right)$, where $p_{T, 1}$ and $p_{T, 2}$ are the transverse momenta of the jets with the largest and second-to-largest transverse momenta. The $A_{J}$ distribution is reasonably broad already in proton-proton collisions, see the black curve in figure 11. The two jets in a dijet need not be back-to-back and need not have the same energy first of all because there may be three or more jets in the event and second of all because of the 


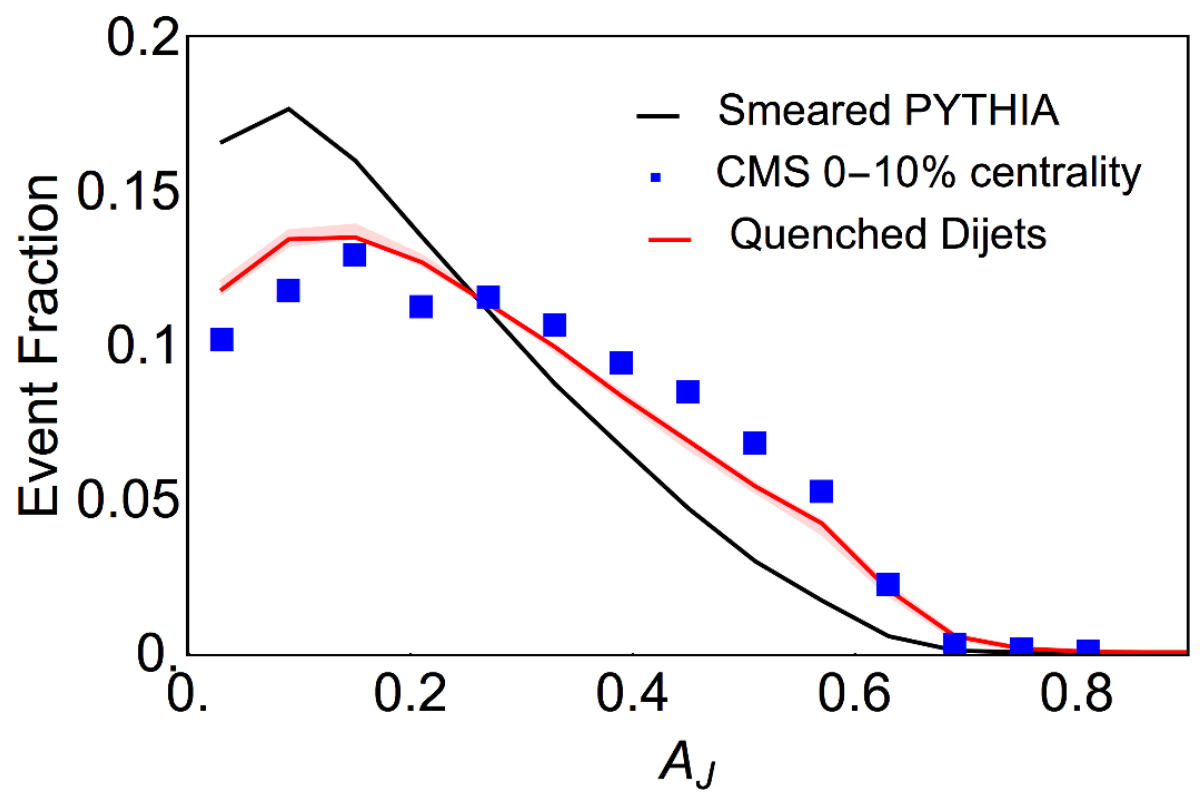

Figure 11. The dijet asymmetry distribution in our ensemble of holographic jets, before quenching (black curve) and after propagation through the strongly-coupled plasma of section 3 (red curve). The red curve is drawn for $a=2$, with the pink band indicating $a=1.8-2.5$. The CMS measurement of the dijet asymmetry distribution in the $0-10 \%$ most central $\mathrm{Pb}-\mathrm{Pb}$ collisions with $\sqrt{s_{N N}}=2.76 \mathrm{TeV}$ from ref. [27] is shown in blue symbols. In order to make a comparison to this data, as we described at the end of section 3 we smeared both the unquenched input distribution that we obtained from PYTHIA and the distribution after quenching that is the output of our calculation, doing the smearing as described in refs. [27, 33],

interplay between the substructure of jets and the algorithms via which jets are found and reconstructed. In heavy ion collisions, jet quenching introduces a significant new source of dijet asymmetry, since one jet in the dijet will always lose more energy as it traverses the plasma than the other. A further broadening of the $A_{J}$ distribution is thus a signature of jet quenching.

The $A_{J}$ distribution in the absence of quenching cannot be captured fully in our model, since we have no analogue of jet finding or reconstruction and since we have no events with more than two jets. What we have done is to construct an ensemble of back-to-back dijets whose $A_{J}$ distribution is as in proton-proton collisions, taking that input distribution from PythiA, and using a half of one of our strings to represent each jet in a dijet pair. We have constructed an ensemble of roughly five million dijet events with a distribution of asymmetries, in addition to the distributions of opening angles, energies, starting positions, and directions within the plasma as in the computation of the jet shape modification. The $A_{J}$ distribution from this ensemble, with the jet energies suitably smeared to take account of the jet energy resolution of the CMS detector, as we described at the end of section 3 , is shown as the black curve in figure 11. Note that although in the analysis reported in figure 11 we have only used back-to-back dijets, we have also constructed a (smaller) ensemble in which we have not assumed that the jets are back-to-back, instead taking the distribution of angles between the jets in dijet events from that measured in ref. [27]. 
This distribution is peaked, favoring jets which are back-to-back, and we have checked that our results for the modification of the distribution of the dijet asymmetry $A_{J}$ are not significantly different in this case than in our (larger) ensemble that is the basis for figure 11, in which the dijets are always formed from a back-to-back pair. What is important is the choice of initial $A_{J}$ distribution.

Next, we send each dijet in the ensemble through its droplet of strongly coupled plasma. Following ref. [27], we then smear the jet energies as described at the end of section 3, select those events in which $p_{T, 1}>120 \mathrm{GeV} / \mathrm{c}$ and $p_{T, 2}>30 \mathrm{GeV} / \mathrm{c}$ after quenching, and compute the $A_{J}$ distribution for this ensemble of dijets that have been quenched via their propagation through the plasma. Our results are shown in red in figure 11, compared with CMS data from the $0-10 \%$ most central $\mathrm{PbPb}$ collisions.

We find qualitative agreement between the modification to the distribution of the dijet asymmetry $A_{J}$ computed in this simple holographic model and heavy ion collision data from CMS. We anticipate that the largest systematic effect not represented in the pink band in figure 11 arises from the absence of three-jet events in our calculation, since these are in fact the origin of much of the dijet asymmetry in proton-proton collisions. We leave the construction of an ensemble of holographic three-jet events to future work.

\section{Acknowledgments}

We thank Francesca Bellini, Jorge Casalderrey-Solana, Paul Chesler, David Chinellato, Yang-Ting Chien, Andrej Ficnar, Alexander Kalweit, Yen-Jie Lee, Simone Marzani, Chris McGinn, Guilherme Milhano, Daniel Pablos and Jesse Thaler for useful discussions. We are especially grateful to Simone Marzani for providing the formulas of ref. [155]. KR acknowledges the hospitality of the CERN Theory Group. AS is partially supported through the LANL/LDRD Program. This work is supported by the U.S. Department of Energy under grant Contract Number DE-SC0011090.

Open Access. This article is distributed under the terms of the Creative Commons Attribution License (CC-BY 4.0), which permits any use, distribution and reproduction in any medium, provided the original author(s) and source are credited.

\section{References}

[1] PHENIX collaboration, K. Adcox et al., Formation of dense partonic matter in relativistic nucleus-nucleus collisions at RHIC: experimental evaluation by the PHENIX collaboration, Nucl. Phys. A 757 (2005) 184 [nucl-ex/0410003] [INSPIRE].

[2] BRAHMS collaboration, I. Arsene et al., Quark gluon plasma and color glass condensate at RHIC? The perspective from the BRAHMS experiment, Nucl. Phys. A 757 (2005) 1 [nucl-ex/0410020] [INSPIRE].

[3] B.B. Back et al., The PHOBOS perspective on discoveries at RHIC, Nucl. Phys. A 757 (2005) 28 [nucl-ex/0410022] [INSPIRE].

[4] STAR collaboration, J. Adams et al., Experimental and theoretical challenges in the search for the quark gluon plasma: the STAR collaboration's critical assessment of the evidence from RHIC collisions, Nucl. Phys. A 757 (2005) 102 [nucl-ex/0501009] [inSPIRE]. 
[5] ALICE collaboration, Elliptic flow of charged particles in $\mathrm{Pb}-\mathrm{Pb}$ collisions at $2.76 \mathrm{TeV}$, Phys. Rev. Lett. 105 (2010) 252302 [arXiv:1011.3914] [INSPIRE].

[6] ATLAS collaboration, Measurement of the pseudorapidity and transverse momentum dependence of the elliptic flow of charged particles in lead-lead collisions at $\sqrt{s_{N N}}=2.76 \mathrm{TeV}$ with the ATLAS detector, Phys. Lett. B 707 (2012) 330 [arXiv:1108.6018] [INSPIRE].

[7] CMS collaboration, Measurement of the elliptic anisotropy of charged particles produced in $\mathrm{Pb}-\mathrm{Pb}$ collisions at $\sqrt{s}_{N N}=2.76 \mathrm{TeV}$, Phys. Rev. C 87 (2013) 014902 [arXiv:1204.1409] [INSPIRE].

[8] D. Teaney, J. Lauret and E.V. Shuryak, Flow at the SPS and RHIC as a quark gluon plasma signature, Phys. Rev. Lett. 86 (2001) 4783 [nucl-th/0011058] [INSPIRE].

[9] P. Huovinen, P.F. Kolb, U.W. Heinz, P.V. Ruuskanen and S.A. Voloshin, Radial and elliptic flow at RHIC: Further predictions, Phys. Lett. B 503 (2001) 58 [hep-ph/0101136] [INSPIRE].

[10] D. Teaney, J. Lauret and E.V. Shuryak, A hydrodynamic description of heavy ion collisions at the SPS and RHIC, nucl-th/0110037 [INSPIRE].

[11] T. Hirano, U.W. Heinz, D. Kharzeev, R. Lacey and Y. Nara, Hadronic dissipative effects on elliptic flow in ultrarelativistic heavy-ion collisions, Phys. Lett. B 636 (2006) 299 [nucl-th/0511046] [INSPIRE].

[12] P. Romatschke and U. Romatschke, Viscosity information from relativistic nuclear collisions: how perfect is the fluid observed at RHIC?, Phys. Rev. Lett. 99 (2007) 172301 [arXiv:0706.1522] [INSPIRE].

[13] M. Luzum and P. Romatschke, Conformal relativistic viscous hydrodynamics: applications to $R H I C$ results at $\sqrt{s_{N N}}=200 \mathrm{GeV}$, Phys. Rev. C 78 (2008) 034915 [Erratum ibid. C 79 (2009) 039903] [arXiv:0804.4015] [INSPIRE].

[14] B. Schenke, S. Jeon and C. Gale, Elliptic and triangular flow in event-by-event $(3+1) D$ viscous hydrodynamics, Phys. Rev. Lett. 106 (2011) 042301 [arXiv:1009.3244] [INSPIRE].

[15] T. Hirano, P. Huovinen and Y. Nara, Elliptic flow in Pb+Pb collisions at $\sqrt{s_{N N}}=2.76$ TeV: hybrid model assessment of the first data, Phys. Rev. C 84 (2011) 011901 [arXiv: 1012.3955] [INSPIRE].

[16] C. Gale, S. Jeon, B. Schenke, P. Tribedy and R. Venugopalan, Event-by-event anisotropic flow in heavy-ion collisions from combined Yang-Mills and viscous fluid dynamics, Phys. Rev. Lett. 110 (2013) 012302 [arXiv: 1209.6330] [INSPIRE].

[17] C. Shen, Z. Qiu, H. Song, J. Bernhard, S. Bass and U. Heinz, The iEBE-VISHNU code package for relativistic heavy-ion collisions, Comput. Phys. Commun. 199 (2016) 61 [arXiv: 1409.8164] [INSPIRE].

[18] C. Shen, J.-F. Paquet, U. Heinz and C. Gale, Photon emission from a momentum anisotropic quark-gluon plasma, Phys. Rev. C 91 (2015) 014908 [arXiv:1410.3404] [INSPIRE].

[19] S. Ryu et al., Importance of the bulk viscosity of QCD in ultrarelativistic heavy-ion collisions, Phys. Rev. Lett. 115 (2015) 132301 [arXiv:1502.01675] [INSPIRE].

[20] J.E. Bernhard et al., Applying Bayesian parameter estimation to relativistic heavy-ion collisions: simultaneous characterization of the initial state and quark-gluon plasma medium, Phys. Rev. C 94 (2016) 024907 [arXiv:1605.03954] [INSPIRE]. 
[21] S.A. Bass, J.E. Bernhard and J.S. Moreland, Determination of quark-gluon-plasma parameters from a global bayesian analysis, Nucl. Phys. A 967 (2017) 67 [arXiv: 1704.07671] [INSPIRE].

[22] G. Policastro, D.T. Son and A.O. Starinets, The shear viscosity of strongly coupled $N=4$ supersymmetric Yang-Mills plasma, Phys. Rev. Lett. 87 (2001) 081601 [hep-th/0104066] [INSPIRE].

[23] A. Buchel and J.T. Liu, Universality of the shear viscosity in supergravity, Phys. Rev. Lett. 93 (2004) 090602 [hep-th/0311175] [INSPIRE].

[24] P. Kovtun, D.T. Son and A.O. Starinets, Viscosity in strongly interacting quantum field theories from black hole physics, Phys. Rev. Lett. 94 (2005) 111601 [hep-th/0405231] [INSPIRE].

[25] ATLAS collaboration, Observation of a centrality-dependent dijet asymmetry in lead-lead collisions at $\sqrt{s_{N N}}=2.77 \mathrm{TeV}$ with the ATLAS detector at the LHC, Phys. Rev. Lett. 105 (2010) 252303 [arXiv: 1011.6182] [INSPIRE].

[26] CMS collaboration, Observation and studies of jet quenching in $\mathrm{PbPb}$ collisions at nucleon-nucleon center-of-mass energy $=2.76$ TeV, Phys. Rev. C 84 (2011) 024906 [arXiv:1102.1957] [INSPIRE].

[27] CMS collaboration, Jet momentum dependence of jet quenching in $\mathrm{PbPb}$ collisions at $\sqrt{s_{N N}}=2.76 \mathrm{TeV}$, Phys. Lett. B 712 (2012) 176 [arXiv:1202.5022] [INSPIRE].

[28] CMS collaboration, Studies of jet quenching using isolated-photon+jet correlations in $\mathrm{PbPb}$ and pp collisions at $\sqrt{s_{N N}}=2.76 \mathrm{TeV}$, Phys. Lett. B 718 (2013) 773 [arXiv:1205.0206] [INSPIRE].

[29] CMS collaboration, Measurement of jet fragmentation into charged particles in pp and $\mathrm{PbPb}$ collisions at $\sqrt{s_{N N}}=2.76 \mathrm{TeV}$, JHEP 10 (2012) 087 [arXiv:1205.5872] [INSPIRE].

[30] ATLAS collaboration, Measurement of the jet radius and transverse momentum dependence of inclusive jet suppression in lead-lead collisions at $\sqrt{s_{N N}}=2.76 \mathrm{TeV}$ with the ATLAS detector, Phys. Lett. B 719 (2013) 220 [arXiv:1208.1967] [INSPIRE].

[31] CMS Collaboration, Nuclear modification factor of high transverse momentum jets in $\mathrm{PbPb}$ collisions at $\sqrt{s_{N N}}=2.76 \mathrm{TeV}$, CMS-PAS-HIN-12-004 (2012).

[32] ATLAS collaboration, Measurement of the azimuthal angle dependence of inclusive jet yields in $\mathrm{Pb}+\mathrm{Pb}$ collisions at $\sqrt{s_{N N}}=2.76 \mathrm{TeV}$ with the ATLAS detector, Phys. Rev. Lett. 111 (2013) 152301 [arXiv: 1306.6469] [INSPIRE].

[33] CMS collaboration, Modification of jet shapes in PbPb collisions at $\sqrt{s_{N N}}=2.76 \mathrm{TeV}$, Phys. Lett. B 730 (2014) 243 [arXiv:1310.0878] [INSPIRE].

[34] ALICE collaboration, Measurement of charged jet suppression in $\mathrm{Pb}-\mathrm{Pb}$ collisions at $\sqrt{s_{N N}}=2.76 \mathrm{TeV}$, JHEP 03 (2014) 013 [arXiv:1311.0633] [INSPIRE].

[35] CMS collaboration, Evidence of b-jet quenching in PbPb collisions at $\sqrt{s_{N N}}=2.76 \mathrm{TeV}$, Phys. Rev. Lett. 113 (2014) 132301 [arXiv:1312.4198] [INSPIRE].

[36] CMS collaboration, Measurement of jet fragmentation in $\mathrm{PbPb}$ and pp collisions at $\sqrt{s_{N N}}=2.76 \mathrm{TeV}$, Phys. Rev. C 90 (2014) 024908 [arXiv: 1406.0932] [INSPIRE].

[37] ATLAS collaboration, Measurement of inclusive jet charged-particle fragmentation functions in $\mathrm{Pb}+\mathrm{Pb}$ collisions at $\sqrt{s_{N N}}=2.76 \mathrm{TeV}$ with the ATLAS detector, Phys. Lett. B 739 (2014) 320 [arXiv:1406.2979] [INSPIRE]. 
[38] ATLAS collaboration, Measurements of the nuclear modification factor for jets in $\mathrm{Pb}+\mathrm{Pb}$ collisions at $\sqrt{s_{N N}}=2.76 \mathrm{TeV}$ with the ATLAS detector, Phys. Rev. Lett. 114 (2015) 072302 [arXiv: 1411.2357] [INSPIRE].

[39] ALICE collaboration, Measurement of jet suppression in central $\mathrm{Pb}$ - $\mathrm{Pb}$ collisions at $\sqrt{s_{N N}}=2.76 \mathrm{TeV}$, Phys. Lett. B 746 (2015) 1 [arXiv: 1502.01689] [inSPIRE].

[40] ALICE collaboration, Measurement of jet quenching with semi-inclusive hadron-jet distributions in central Pb-Pb collisions at $\sqrt{s_{N N}}=2.76 \mathrm{TeV}$, JHEP 09 (2015) 170 [arXiv: 1506.03984] [INSPIRE].

[41] ATLAS collaboration, Measurement of the production of neighbouring jets in lead-lead collisions at $\sqrt{s_{N N}}=2.76 \mathrm{TeV}$ with the ATLAS detector, Phys. Lett. B 751 (2015) 376 [arXiv: 1506.08656] [INSPIRE].

[42] CMS Collaboration, Study of isolated-photon + jet correlations in PbPb and pp collisions at $\sqrt{s_{N N}}=5.02 \mathrm{TeV}$, CMS-PAS-HIN-16-002 (2016).

[43] CMS collaboration, Measurement of transverse momentum relative to dijet systems in $\mathrm{PbPb}$ and pp collisions at $\sqrt{s_{N N}}=2.76 \mathrm{TeV}$, JHEP 01 (2016) 006 [arXiv: 1509.09029] [INSPIRE].

[44] CMS collaboration, Correlations between jets and charged particles in $\mathrm{PbPb}$ and $p p$ collisions at $\sqrt{s_{N N}}=2.76 \mathrm{TeV}$, JHEP 02 (2016) 156 [arXiv:1601.00079] [INSPIRE].

[45] CMS collaboration, Decomposing transverse momentum balance contributions for quenched jets in PbPb collisions at $\sqrt{s_{N N}}=2.76 \mathrm{TeV}$, JHEP 11 (2016) 055 [arXiv:1609.02466] [INSPIRE].

[46] CMS collaboration, Measurement of inclusive jet cross sections in $p p$ and $\mathrm{PbPb}$ collisions at $\sqrt{s_{N N}}=2.76 \mathrm{TeV}$, Phys. Rev. C 96 (2017) 015202 [arXiv: 1609.05383] [INSPIRE].

[47] ATLAS collaboration, Measurement of jet fragmentation in $\mathrm{Pb}+\mathrm{Pb}$ and pp collisions at $\sqrt{s_{N N}}=2.76 \mathrm{TeV}$ with the ATLAS detector at the LHC, Eur. Phys. J. C 77 (2017) 379 [arXiv: 1702.00674] [INSPIRE].

[48] ALICE collaboration, First measurement of jet mass in $P b-P b$ and $p-P b$ collisions at the LHC, Phys. Lett. B 776 (2018) 249 [arXiv:1702.00804] [INSPIRE].

[49] CMS collaboration, Study of jet quenching with $Z+$ jet correlations in $P b-P b$ and $p p$ collisions at $\sqrt{s_{N N}}=5.02 \mathrm{TeV}$, Phys. Rev. Lett. 119 (2017) 082301 [arXiv:1702.01060] [INSPIRE].

[50] ATLAS collaboration, Measurement of jet $p_{T}$ correlations in $\mathrm{Pb}+\mathrm{Pb}$ and $\mathrm{pp}$ collisions at $\sqrt{s_{N N}}=2.76 \mathrm{TeV}$ with the ATLAS detector, Phys. Lett. B 774 (2017) 379 [arXiv: 1706.09363] [INSPIRE].

[51] PHENIX collaboration, K. Adcox et al., Suppression of hadrons with large transverse momentum in central Au+Au collisions at $\sqrt{s_{N N}}=130 \mathrm{GeV}$, Phys. Rev. Lett. 88 (2002) 022301 [nucl-ex/0109003] [INSPIRE].

[52] STAR collaboration, C. Adler et al., Centrality dependence of high $p_{T}$ hadron suppression in Au+Au collisions at $\sqrt{s}_{N N}=130 \mathrm{GeV}$, Phys. Rev. Lett. 89 (2002) 202301 [nucl-ex/0206011] [INSPIRE].

[53] STAR collaboration, C. Adler et al., Disappearance of back-to-back high $p_{T}$ hadron correlations in central Au+Au collisions at $\sqrt{s_{N N}}=200 \mathrm{GeV}$, Phys. Rev. Lett. 90 (2003) 082302 [nucl-ex/0210033] [INSPIRE]. 
[54] STAR collaboration, M. Ploskon, Inclusive cross section and correlations of fully reconstructed jets in $\sqrt{s_{N N}}=200 \mathrm{GEV} A u+A u$ and $p+p$ collisions, Nucl. Phys. A 830 (2009) 255C-258C [arXiv:0908.1799] [INSPIRE].

[55] PHENIX collaboration, D.V. Perepelitsa, Reconstructed jet results in $p+p, d+$ Au and Cu + Cu collisions at 200 GeV from PHENIX, Nucl. Phys. A 910-911 (2013) 425.

[56] STAR collaboration, L. Adamczyk et al., Jet-Hadron correlations in $\sqrt{s_{N N}}=200 \mathrm{GeV}$ $p+p$ and central Au+Au collisions, Phys. Rev. Lett. 112 (2014) 122301 [arXiv:1302.6184] [INSPIRE].

[57] STAR collaboration, P.M. Jacobs and A. Schmah, Measurements of jet quenching with semi-inclusive charged jet distributions in Au+Au collisions at $\sqrt{s_{N N}}=200 \mathrm{GeV}$, Nucl. Phys. A 956 (2016) 641 [arXiv:1512.08784] [INSPIRE].

[58] STAR collaboration, L. Adamczyk et al., Dijet imbalance measurements in Au+Au and pp collisions at $\sqrt{s_{N N}}=200 \mathrm{GeV}$ at STAR, Phys. Rev. Lett. 119 (2017) 062301 [arXiv: 1609.03878] [INSPIRE].

[59] STAR collaboration, L. Adamczyk et al., Measurements of jet quenching with semi-inclusive hadron + jet distributions in Au+Au collisions at $\sqrt{s_{N N}}=200 \mathrm{GeV}$, Phys. Rev. C 96 (2017) 024905 [arXiv: 1702.01108] [INSPIRE].

[60] A. Adare et al., An upgrade proposal from the PHENIX collaboration, arXiv:1501.06197 [INSPIRE].

[61] P. Jacobs and X.-N. Wang, Matter in extremis: ultrarelativistic nuclear collisions at RHIC, Prog. Part. Nucl. Phys. 54 (2005) 443 [hep-ph/0405125] [INSPIRE].

[62] J. Casalderrey-Solana and C.A. Salgado, Introductory lectures on jet quenching in heavy ion collisions, Acta Phys. Polon. B 38 (2007) 3731 [arXiv:0712.3443] [InSPIRE].

[63] A. Majumder and M. Van Leeuwen, The theory and phenomenology of perturbative QCD based jet quenching, Prog. Part. Nucl. Phys. 66 (2011) 41 [arXiv:1002.2206] [INSPIRE].

[64] Y. Mehtar-Tani, J.G. Milhano and K. Tywoniuk, Jet physics in heavy-ion collisions, Int. J. Mod. Phys. A 28 (2013) 1340013 [arXiv:1302.2579] [InSPIRE].

[65] J. Ghiglieri and D. Teaney, Parton energy loss and momentum broadening at NLO in high temperature QCD plasmas, Int. J. Mod. Phys. E 24 (2015) 1530013 [arXiv:1502.03730] [INSPIRE].

[66] J.-P. Blaizot and Y. Mehtar-Tani, Jet structure in heavy ion collisions, Int. J. Mod. Phys. E 24 (2015) 1530012 [arXiv: 1503.05958] [INSPIRE].

[67] G.-Y. Qin and X.-N. Wang, Jet quenching in high-energy heavy-ion collisions, Int. J. Mod. Phys. E 24 (2015) 1530014 [arXiv:1511.00790] [InSPIRE].

[68] K. Zapp, J. Stachel and U.A. Wiedemann, A local Monte Carlo implementation of the non-abelian Landau-Pomerantschuk-Migdal effect, Phys. Rev. Lett. 103 (2009) 152302 [arXiv:0812.3888] [INSPIRE].

[69] K. Zapp, G. Ingelman, J. Rathsman, J. Stachel and U.A. Wiedemann, A Monte Carlo model for 'jet quenching', Eur. Phys. J. C 60 (2009) 617 [arXiv:0804.3568] [INSPIRE].

[70] N. Armesto, L. Cunqueiro and C.A. Salgado, Q-PYTHIA: a medium-modified implementation of final state radiation, Eur. Phys. J. C 63 (2009) 679 [arXiv:0907.1014] [INSPIRE].

[71] B. Schenke, C. Gale and S. Jeon, MARTINI: an event generator for relativistic heavy-ion collisions, Phys. Rev. C 80 (2009) 054913 [arXiv:0909. 2037] [INSPIRE]. 
[72] I.P. Lokhtin, A.V. Belyaev and A.M. Snigirev, Jet quenching pattern at LHC in PYQUEN model, Eur. Phys. J. C 71 (2011) 1650 [arXiv:1103.1853] [INSPIRE].

[73] K.C. Zapp, F. Krauss and U.A. Wiedemann, A perturbative framework for jet quenching, JHEP 03 (2013) 080 [arXiv:1212.1599] [InSPIRE].

[74] K.C. Zapp, JEWEL 2.0.0: directions for use, Eur. Phys. J. C 74 (2014) 2762 [arXiv: 1311.0048] [INSPIRE].

[75] K.C. Zapp, Geometrical aspects of jet quenching in JEWEL, Phys. Lett. B 735 (2014) 157 [arXiv: 1312.5536] [INSPIRE].

[76] JETSCAPE collaboration, S. Cao et al., Multistage Monte-Carlo simulation of jet modification in a static medium, Phys. Rev. C 96 (2017) 024909 [arXiv:1705.00050] [INSPIRE].

[77] I. Vitev and B.-W. Zhang, Jet tomography of high-energy nucleus-nucleus collisions at next-to-leading order, Phys. Rev. Lett. 104 (2010) 132001 [arXiv:0910.1090] [INSPIRE].

[78] J. Casalderrey-Solana, J.G. Milhano and U.A. Wiedemann, Jet quenching via jet collimation, J. Phys. G 38 (2011) 035006 [arXiv:1012.0745] [INSPIRE].

[79] G.-Y. Qin and B. Müller, Explanation of di-jet asymmetry in Pb+Pb collisions at the Large Hadron Collider, Phys. Rev. Lett. 106 (2011) 162302 [Erratum ibid. 108 (2012) 189904] [arXiv: 1012.5280] [INSPIRE].

[80] C. Young, B. Schenke, S. Jeon and C. Gale, Dijet asymmetry at the energies available at the CERN Large Hadron Collider, Phys. Rev. C 84 (2011) 024907 [arXiv:1103.5769] [INSPIRE].

[81] Y. He, I. Vitev and B.-W. Zhang, $\mathcal{O}\left(\alpha_{s}^{3}\right)$ analysis of inclusive jet and di-jet production in heavy ion reactions at the large hadron collider, Phys. Lett. B 713 (2012) 224 [arXiv:1105.2566] [INSPIRE].

[82] J. Casalderrey-Solana, J.G. Milhano and U. Wiedemann, Jet quenching via jet collimation, J. Phys. G 38 (2011) 124086 [arXiv:1107.1964] [InSPIRE].

[83] T. Renk, On the sensitivity of the dijet asymmetry to the physics of jet quenching, Phys. Rev. C 85 (2012) 064908 [arXiv: 1202.4579] [INSPIRE].

[84] R.B. Neufeld and I. Vitev, The $Z^{0}$-tagged jet event asymmetry in heavy-ion collisions at the CERN Large Hadron Collider, Phys. Rev. Lett. 108 (2012) 242001 [arXiv:1202.5556] [INSPIRE].

[85] T. Renk, Energy dependence of the dijet imbalance in $\mathrm{Pb}-\mathrm{Pb}$ collisions at 2.76 ATeV, Phys. Rev. C 86 (2012) 061901 [arXiv: 1204.5572] [INSPIRE].

[86] W. Dai, I. Vitev and B.-W. Zhang, Momentum imbalance of isolated photon-tagged jet production at RHIC and LHC, Phys. Rev. Lett. 110 (2013) 142001 [arXiv:1207.5177] [INSPIRE].

[87] L. Apolinario, N. Armesto and L. Cunqueiro, An analysis of the influence of background subtraction and quenching on jet observables in heavy-ion collisions, JHEP 02 (2013) 022 [arXiv:1211.1161] [INSPIRE].

[88] X.-N. Wang and Y. Zhu, Medium modification of $\gamma$-jets in high-energy heavy-ion collisions, Phys. Rev. Lett. 111 (2013) 062301 [arXiv:1302.5874] [INSPIRE].

[89] G.-L. Ma, Dijet asymmetry in $P b+P b$ collisions at $\sqrt{s_{N N}}=2.76 \mathrm{TeV}$ within a multiphase transport model, Phys. Rev. C 87 (2013) 064901 [arXiv: 1304.2841] [INSPIRE]. 
[90] J. Huang, Z.-B. Kang and I. Vitev, Inclusive b-jet production in heavy ion collisions at the LHC, Phys. Lett. B 726 (2013) 251 [arXiv:1306.0909] [inSPIRE].

[91] F. Senzel, O. Fochler, J. Uphoff, Z. Xu and C. Greiner, Influence of multiple in-medium scattering processes on the momentum imbalance of reconstructed di-jets, J. Phys. G 42 (2015) 115104 [arXiv:1309.1657] [InSPIRE].

[92] R. Perez-Ramos and T. Renk, In-medium jet shape from energy collimation in parton showers: comparison with CMS PbPb data at 2.76 TeV, Phys. Rev. D 90 (2014) 014018 [arXiv: 1401.5283] [INSPIRE].

[93] T. Renk, A study of the constraining power of high $P_{T}$ observables in heavy-ion collisions, arXiv: 1408.6684 [INSPIRE].

[94] R. Pérez-Ramos and T. Renk, A Monte Carlo study of jet fragmentation functions in $\mathrm{PbPb}$ and $p p$ collisions at $\sqrt{s}=2.76 \mathrm{TeV}$, arXiv:1411.1983 [INSPIRE].

[95] Y.-T. Chien, A. Emerman, Z.-B. Kang, G. Ovanesyan and I. Vitev, Jet quenching from QCD evolution, Phys. Rev. D 93 (2016) 074030 [arXiv:1509.02936] [INSPIRE].

[96] J. Huang, Z.-B. Kang, I. Vitev and H. Xing, Photon-tagged and B-meson-tagged b-jet production at the LHC, Phys. Lett. B 750 (2015) 287 [arXiv:1505.03517] [INSPIRE].

[97] Y.-T. Chien and I. Vitev, Towards the understanding of jet shapes and cross sections in heavy ion collisions using soft-collinear effective theory, JHEP 05 (2016) 023 [arXiv: 1509.07257] [INSPIRE].

[98] J.G. Milhano and K.C. Zapp, Origins of the di-jet asymmetry in heavy ion collisions, Eur. Phys. J. C 76 (2016) 288 [arXiv:1512.08107] [INSPIRE].

[99] X. Zhang, L. Apolinário, J.G. Milhano and M. Płoskoń, Sub-jet structure as a discriminating quenching probe, Nucl. Phys. A 956 (2016) 597 [arXiv:1512.09255] [INSPIRE].

[100] N.-B. Chang and G.-Y. Qin, Full jet evolution in quark-gluon plasma and nuclear modification of jet production and jet shape in $\mathrm{Pb}+\mathrm{Pb}$ collisions at 2.76 ATeV at the CERN Large Hadron Collider, Phys. Rev. C 94 (2016) 024902 [arXiv:1603.01920] [InSPIRE].

[101] A.H. Mueller, B. Wu, B.-W. Xiao and F. Yuan, Probing transverse momentum broadening in heavy ion Collisions, Phys. Lett. B 763 (2016) 208 [arXiv: 1604.04250] [InSPIRE].

[102] L. Chen, G.-Y. Qin, S.-Y. Wei, B.-W. Xiao and H.-Z. Zhang, Probing transverse momentum broadening via dihadron and hadron-jet angular correlations in relativistic heavy-ion collisions, Phys. Lett. B 773 (2017) 672 [arXiv:1607.01932] [INSPIRE].

[103] Y. Mehtar-Tani and K. Tywoniuk, Groomed jets in heavy-ion collisions: sensitivity to medium-induced bremsstrahlung, JHEP 04 (2017) 125 [arXiv: 1610.08930] [INSPIRE].

[104] Y. Tachibana, N.-B. Chang and G.-Y. Qin, Full jet in quark-gluon plasma with hydrodynamic medium response, Phys. Rev. C 95 (2017) 044909 [arXiv:1701.07951] [INSPIRE].

[105] R. Kunnawalkam Elayavalli and K.C. Zapp, Medium response in JEWEL and its impact on jet shape observables in heavy ion collisions, JHEP 07 (2017) 141 [arXiv:1707.01539] [INSPIRE].

[106] J.G. Milhano, U.A. Wiedemann and K.C. Zapp, Sensitivity of jet substructure to jet-induced medium response, arXiv:1707.04142 [INSPIRE].

[107] Y. Mehtar-Tani and K. Tywoniuk, Quenching of high-p $p_{T}$ jet spectra, arXiv:1707.07361 [INSPIRE]. 
[108] J.M. Maldacena, The large-N limit of superconformal field theories and supergravity, Int. J. Theor. Phys. 38 (1999) 1113 [hep-th/9711200] [InSPIRE].

[109] J. Casalderrey-Solana, H. Liu, D. Mateos, K. Rajagopal and U.A. Wiedemann, Gauge/string duality, hot QCD and heavy ion collisions, arXiv:1101.0618 [INSPIRE].

[110] O. DeWolfe, S.S. Gubser, C. Rosen and D. Teaney, Heavy ions and string theory, Prog. Part. Nucl. Phys. 75 (2014) 86 [arXiv:1304.7794] [InSPIRE].

[111] P.M. Chesler and W. van der Schee, Early thermalization, hydrodynamics and energy loss in AdS/CFT, Int. J. Mod. Phys. E 24 (2015) 1530011 [arXiv:1501.04952] [InSPIRE].

[112] C.P. Herzog, A. Karch, P. Kovtun, C. Kozcaz and L.G. Yaffe, Energy loss of a heavy quark moving through $N=4$ supersymmetric Yang-Mills plasma, JHEP 07 (2006) 013 [hep-th/0605158] [INSPIRE].

[113] H. Liu, K. Rajagopal and U.A. Wiedemann, Calculating the jet quenching parameter from AdS/CFT, Phys. Rev. Lett. 97 (2006) 182301 [hep-ph/0605178] [INSPIRE].

[114] J. Casalderrey-Solana and D. Teaney, Heavy quark diffusion in strongly coupled $N=4$ Yang-Mills, Phys. Rev. D 74 (2006) 085012 [hep-ph/0605199] [InSPIRE].

[115] S.S. Gubser, Drag force in AdS/CFT, Phys. Rev. D 74 (2006) 126005 [hep-th/0605182] [INSPIRE].

[116] H. Liu, K. Rajagopal and U.A. Wiedemann, An AdS/CFT calculation of screening in a hot wind, Phys. Rev. Lett. 98 (2007) 182301 [hep-ph/0607062] [INSPIRE].

[117] H. Liu, K. Rajagopal and U.A. Wiedemann, Wilson loops in heavy ion collisions and their calculation in AdS/CFT, JHEP 03 (2007) 066 [hep-ph/0612168] [INSPIRE].

[118] S.S. Gubser, Momentum fluctuations of heavy quarks in the gauge-string duality, Nucl. Phys. B 790 (2008) 175 [hep-th/0612143] [INSPIRE].

[119] M. Chernicoff, J.A. Garcia and A. Guijosa, The energy of a moving quark-antiquark pair in an $N=4$ SYM plasma, JHEP 09 (2006) 068 [hep-th/0607089] [INSPIRE].

[120] J. Casalderrey-Solana and D. Teaney, Transverse momentum broadening of a fast quark in a $N=4$ Yang-Mills plasma, JHEP 04 (2007) 039 [hep-th/0701123] [INSPIRE].

[121] P.M. Chesler and L.G. Yaffe, The Wake of a quark moving through a strongly-coupled plasma, Phys. Rev. Lett. 99 (2007) 152001 [arXiv:0706.0368] [INSPIRE].

[122] S.S. Gubser, S.S. Pufu and A. Yarom, Sonic booms and diffusion wakes generated by a heavy quark in thermal AdS/CFT, Phys. Rev. Lett. 100 (2008) 012301 [arXiv:0706.4307] [INSPIRE].

[123] P.M. Chesler and L.G. Yaffe, The stress-energy tensor of a quark moving through a strongly-coupled $N=4$ supersymmetric Yang-Mills plasma: Comparing hydrodynamics and AdS/CFT, Phys. Rev. D 78 (2008) 045013 [arXiv:0712.0050] [INSPIRE].

[124] D.M. Hofman and J. Maldacena, Conformal collider physics: Energy and charge correlations, JHEP 05 (2008) 012 [arXiv:0803.1467] [INSPIRE].

[125] S.S. Gubser, D.R. Gulotta, S.S. Pufu and F.D. Rocha, Gluon energy loss in the gauge-string duality, JHEP 10 (2008) 052 [arXiv: 0803.1470] [INSPIRE].

[126] Y. Hatta, E. Iancu and A.H. Mueller, Jet evolution in the $N=4$ SYM plasma at strong coupling, JHEP 05 (2008) 037 [arXiv:0803.2481] [INSPIRE]. 
[127] F. Dominguez, C. Marquet, A.H. Mueller, B. Wu and B.-W. Xiao, Comparing energy loss and p-perpendicular-broadening in perturbative QCD with strong coupling $N=4 S Y M$ theory, Nucl. Phys. A 811 (2008) 197 [arXiv:0803.3234] [inSPIRE].

[128] P.M. Chesler, K. Jensen and A. Karch, Jets in strongly-coupled $N=4$ super Yang-Mills theory, Phys. Rev. D 79 (2009) 025021 [arXiv:0804.3110] [InSPIRE].

[129] P.M. Chesler, K. Jensen, A. Karch and L.G. Yaffe, Light quark energy loss in strongly-coupled $N=4$ supersymmetric Yang-Mills plasma, Phys. Rev. D 79 (2009) 125015 [arXiv:0810.1985] [INSPIRE].

[130] F. D'Eramo, H. Liu and K. Rajagopal, Transverse momentum broadening and the jet quenching parameter, redux, Phys. Rev. D 84 (2011) 065015 [arXiv:1006.1367] [INSPIRE].

[131] P. Arnold and D. Vaman, Jet quenching in hot strongly coupled gauge theories revisited: 3-point correlators with gauge-gravity duality, JHEP 10 (2010) 099 [arXiv: 1008.4023] [INSPIRE].

[132] P. Arnold and D. Vaman, Jet quenching in hot strongly coupled gauge theories simplified, JHEP 04 (2011) 027 [arXiv:1101.2689] [InSPIRE].

[133] P. Arnold and D. Vaman, Some new results for 'jet' stopping in AdS/CFT: long version, J. Phys. G 38 (2011) 124175 [arXiv: 1106.1680] [INSPIRE].

[134] M. Chernicoff, J.A. Garcia, A. Guijosa and J.F. Pedraza, Holographic lessons for quark dynamics, J. Phys. G 39 (2012) 054002 [arXiv:1111.0872] [INSPIRE].

[135] P.M. Chesler, Y.-Y. Ho and K. Rajagopal, Shining a gluon beam through quark-gluon plasma, Phys. Rev. D 85 (2012) 126006 [arXiv:1111.1691] [InSPIRE].

[136] P. Arnold, P. Szepietowski and D. Vaman, Coupling dependence of jet quenching in hot strongly-coupled gauge theories, JHEP 07 (2012) 024 [arXiv: 1203.6658] [INSPIRE].

[137] P. Arnold, P. Szepietowski, D. Vaman and G. Wong, Tidal stretching of gravitons into classical strings: application to jet quenching with AdS/CFT, JHEP 02 (2013) 130 [arXiv: 1212.3321] [INSPIRE].

[138] P.M. Chesler, M. Lekaveckas and K. Rajagopal, Heavy quark energy loss far from equilibrium in a strongly coupled collision, JHEP 10 (2013) 013 [arXiv: 1306.0564] [INSPIRE].

[139] A. Ficnar and S.S. Gubser, Finite momentum at string endpoints, Phys. Rev. D 89 (2014) 026002 [arXiv: 1306.6648] [INSPIRE].

[140] A. Ficnar, S.S. Gubser and M. Gyulassy, Shooting string holography of jet quenching at RHIC and LHC, Phys. Lett. B 738 (2014) 464 [arXiv:1311.6160] [INSPIRE].

[141] P.M. Chesler and K. Rajagopal, Jet quenching in strongly coupled plasma, Phys. Rev. D 90 (2014) 025033 [arXiv:1402.6756] [INSPIRE].

[142] R. Rougemont, A. Ficnar, S. Finazzo and J. Noronha, Energy loss, equilibration and thermodynamics of a baryon rich strongly coupled quark-gluon plasma, JHEP 04 (2016) 102 [arXiv: 1507.06556] [INSPIRE].

[143] P.M. Chesler and K. Rajagopal, On the evolution of jet energy and opening angle in strongly coupled plasma, JHEP 05 (2016) 098 [arXiv: 1511.07567] [INSPIRE].

[144] J. Casalderrey-Solana and A. Ficnar, Holographic three-jet events in strongly coupled $N=4$ Yang-Mills plasma, arXiv:1512.00371 [INSPIRE]. 
[145] K. Rajagopal, A.V. Sadofyev and W. van der Schee, Evolution of the jet opening angle distribution in holographic plasma, Phys. Rev. Lett. 116 (2016) 211603 [arXiv:1602.04187] [INSPIRE].

[146] J. Brewer, K. Rajagopal, A. Sadofyev and W. van der Schee, Holographic jet shapes and their evolution in strongly coupled plasma, Nucl. Phys. A 967 (2017) 508 [arXiv: 1704.05455] [INSPIRE].

[147] J. Casalderrey-Solana et al., A hybrid strong/weak coupling approach to jet quenching, JHEP 10 (2014) 019 [Erratum ibid. 09 (2015) 175] [arXiv:1405. 3864] [INSPIRE].

[148] J. Casalderrey-Solana et al., Predictions for boson-jet observables and fragmentation function ratios from a hybrid strong/weak coupling model for jet quenching, JHEP 03 (2016) 053 [arXiv: 1508.00815] [INSPIRE].

[149] J. Casalderrey-Solana et al., Angular structure of jet quenching within a hybrid strong/weak coupling model, JHEP 03 (2017) 135 [arXiv:1609.05842] [INSPIRE].

[150] Z. Hulcher, D. Pablos and K. Rajagopal, Resolution effects in the hybrid strong/weak coupling model, arXiv: 1707.05245 [INSPIRE].

[151] A. Karch and E. Katz, Adding flavor to AdS/CFT, JHEP 06 (2002) 043 [hep-th/0205236] [INSPIRE].

[152] R. Morad and W.A. Horowitz, Strong-coupling jet energy loss from AdS/CFT, JHEP 11 (2014) 017 [arXiv: 1409.7545] [InSPIRE].

[153] S.S. Gubser, Comparing the drag force on heavy quarks in $N=4$ super-Yang-Mills theory and QCD, Phys. Rev. D 76 (2007) 126003 [hep-th/0611272] [INSPIRE].

[154] M. Cacciari, G.P. Salam and G. Soyez, The anti-k $k_{t}$ jet clustering algorithm, JHEP 04 (2008) 063 [arXiv: 0802.1189] [INSPIRE].

[155] A.J. Larkoski, S. Marzani, G. Soyez and J. Thaler, Soft drop, JHEP 05 (2014) 146 [arXiv: 1402.2657] [INSPIRE].

[156] ALICE collaboration, Centrality dependence of the pseudorapidity density distribution for charged particles in Pb-Pb collisions at $\sqrt{s_{\mathrm{NN}}}=2.76 \mathrm{TeV}$, Phys. Lett. B 726 (2013) 610 [arXiv: 1304.0347] [INSPIRE].

[157] ALICE collaboration, Centrality dependence of $\pi, K, p$ production in $P b-P b$ collisions at $\sqrt{s_{N N}}=2.76 \mathrm{TeV}$, Phys. Rev. C 88 (2013) 044910 [arXiv: 1303.0737] [INSPIRE].

[158] M. Habich, J.L. Nagle and P. Romatschke, Particle spectra and HBT radii for simulated central nuclear collisions of $C+C, A l+A l, C u+C u, A u+A u$ and $P b+P b$ from $\sqrt{s}=62.4-2760 \mathrm{GeV}$, Eur. Phys. J. C 75 (2015) 15 [arXiv:1409.0040] [inSPIRE].

[159] K. Rajagopal and A.V. Sadofyev, Chiral drag force, JHEP 10 (2015) 018 [arXiv: 1505. 07379] [INSPIRE]. 